\title{
Development of Modeling Capabilities for Launch Pad Acoustics and Ignition Transient Environment Prediction
}

\author{
Jeff West ${ }^{1}$ and Louise L. Strutzenberg ${ }^{2}$ \\ NASA/Marshall Space Flight Center, Huntsville, Alabama, USA \\ Gabriel C. Putnam ${ }^{3}$ \\ All Points Logistics, Huntsville, Alabama, USA \\ Peter A. Liever ${ }^{4}$, and Brandon R. Williams ${ }^{5}$ \\ CFD Research Corporation (CFDRC), Huntsville, Alabama, USA
}

This paper presents the development effort to establish modeling capabilities for launch vehicle liftoff acoustics and ignition transient environment predictions. Peak acoustic loads experienced by the launch vehicle occur during liftoff with strong interaction between the vehicle and the launch facility. Acoustic prediction engineering tools based on empirical models are of limited value in efforts to proactively design and optimize launch vehicles and launch facility configurations for liftoff acoustics. Modeling approaches are needed that capture the important details of the plume flow environment including the ignition transient, identify the noise generation sources and allow assessment of the effects of launch pad geometric details and acoustic mitigation measures such as water injection. This paper will present a status of the CFD tools developed by the Marshall Space Flight Center (MSFC) Fluid Dynamics Branch featuring relevant advanced multi-physics modeling capabilities and related efforts to establish a hybrid acoustic environment modeling capability combining CFD with a Boundary Element Method (BEM) acoustic field propagation model.

The CFD analysis software program utilized at the MSFC Fluid Dynamics Branch is Loci/CHEM. Loci is a highly scalable computational framework with automatic parallelization for computational field simulations [1, 2]. Loci was developed at Mississippi State University by Dr. Ed Luke. Loci/CHEM is a density-based Navier-Stokes solver [3] implemented in the Loci framework with the following features:

- Generalized unstructured grids

- RANS, URANS, DES, Hybrid RANS/LES turbulence modeling

- Eulerian multiphase models for particulates and droplets

- Lagrangian multiphase models for particulates and droplets with particle vaporization, condensation, combustion

- Real fluids EOS for cryogenic injection and combustion analysis

- Non-gray radiation transport models (particle and gas phase radiation)

- Solution adaptive mesh refinement with various error estimators available

- Mesh deformation for fluid-structure deformation and fuel burn-back surface

- Overset moving body with prescribed motion and 6-DOF

- Body Collision 6-DOF modeling

Loci/CHEM has been extensively verified using the Method of Manufactured Solutions (MMS) Technique. The numerical algorithms are verified to be $2^{\text {nd }}$ order space and $2^{\text {nd }}$ order time accurate, but do not feature low dispersion and low dissipation algorithms at this point of development. Production simulations are routinely executed with up to $300 \mathrm{M}$ mesh cells on more than 3000 processors on the NASA NAS Pleiades supercomputer.

\footnotetext{
${ }^{1}$ AST Fluid Mechanics, Fluid Dynamics Branch/ER42, AIAA Member.

${ }^{2}$ AST Fluid Mechanics, Fluid Dynamics Branch/ER42, AIAA Member.

${ }^{3}$ Engineer, Fluid Dynamics

${ }^{4}$ Technical Fellow, AIAA Senior Member.

${ }^{5}$ Senior Engineer, AIAA Member.
} 
The advances in CFD simulation capability and fidelity in the Fluid Dynamics Branch at MSFC have resulted in CFD modeling of complete launch vehicles with multiple plumes interacting with detailed launch pad geometric models (Figure 1). These analyses have become invaluable in defining liftoff environments for Shuttle and future NASA heavy lift launch vehicle designs. Simulations with these tools are capable of capturing the sources of acoustic waves originating from the plumes and the plume interaction with the facility structures. Advanced multiphase modeling capabilities such as the injection of deluge water in the CFD model have been added recently that enable the assessment of deluge water in affecting the acoustic source regions (Figures 2 and 3). These capabilities now offer the possibility of analysis support for the geometric design of the launch pad and targeted placement of water injection systems.

A CFD model of the acoustic environments due to solid rocket motor ignition transients has been successfully validated, providing significant evidence that CFD models of this class are capable of capturing the sources of acoustic waves originating from the plumes and the plume interaction with the launch pad structures. The Ares I Scale Model Acoustics Test (ASMAT) is a series of live-fire tests of scaled rocket motors performed at MSFC [4]. These tests were designed to simulate the conditions of the Ares I launch configuration. These tests were heavily instrumented and provided a well documented set of high fidelity acoustic measurements useful for validation including data taken over a range of test conditions and containing phenomena like Ignition Over-Pressure and water suppression of acoustics. To take advantage of this data, a computational model of the ASMAT test setup has been constructed and test firings of the motor have been simulated using the Loci/CHEM computational fluid dynamics software (Figure 4). Results from ASMAT simulations with the rocket in both held down and elevated configurations, as well as with and without water suppression have been compared to acoustic data collected from similar live-fire tests [5]. Results of acoustic field predictions with the CFD models have shown good correlation with the amplitude and temporal shape of pressure features and reasonable spectral accuracy up to approximately $1000 \mathrm{~Hz}$. Major plume and acoustic features have been well captured including the plume shock structure, the igniter pulse transient, and the ignition overpressure.

The primary figure of merit for the design of an effective combination of launch pad geometry and water deluge system is the resulting acoustic load reaching the launch vehicle payload. The CFD methodologies developed capture the relevant physics of multi-species plumes with multi-phase modeling of water injection into the plume flow field. They are, however, numerically too dissipative and are not suitable to accurately capture the propagation of the acoustic waves from the plumes and the launch pad impingement regions to the top of the vehicle. A hybrid approach is being developed that combines the high-fidelity CFD analysis capable of identifying the acoustic sources with a Boundary Element Method (BEM) that accurately propagates the acoustic field from the source locations.

The implementation of a BEM method for hybrid CFD/CAA will be presented. This process is facilitated through a NASA Small Business Technology Transfer project (STTR) by the team of CFD Research Corporation and the University of Cincinnati to utilize technology transfer from existing software. The FastBEM Acoustics software was originally developed by Prof. Y. Liu at the University of Cincinnati provides the BEM capability [6]. FastBEM solves the Helmholtz Equation via Boundary Integral Equation (BIE) form. It uses a fast multi-pole (FMM) approach for solving 3-D, interior/exterior, radiation/scattering problems with velocity, pressure and impedance boundary conditions. The Fast Multi-pole Method (FMM) reduces the cost (CPU time \& storage) for BEM to $O(N)$. Large-scale acoustic BEM models with unknowns (DOFs) up to several millions have been solved on desktop workstations. Extension to larger models resulting from the large and complex topology of a launch vehicle and complete launch pad will likely require the parallelization and porting of the software to NASA supercomputer systems.

The technology implementation process involves several steps. The first steps is to extract acoustics source surfaces enclosing acoustic noise source regions (Figure 5). Enclosing surfaces resulting from plume interaction with launch pad structures are considerably more complex than for relatively simple free jet acoustics simulations. Various approaches to this problem are being evaluated. The process of extracting and passing the acoustic source strength information from the enclosure surface to FastBEM input BC are being implemented utilizing existing Loci/CHEM native post-processing tools. The technology transfer process and examples of FastBEM software application to analyze the complex launch pad topology with launch vehicle plume acoustic sources will be presented. Details on the process development and validations against standard acoustic experiments (supersonic plumes, etc.) will be 
presented. Plans for modifications and improvements of the technology for NASA specific applications will be presented, in particular the parallelization and installation of the software system on NASA supercomputer systems to process the larger simulations resulting from complete launch pad configurations.

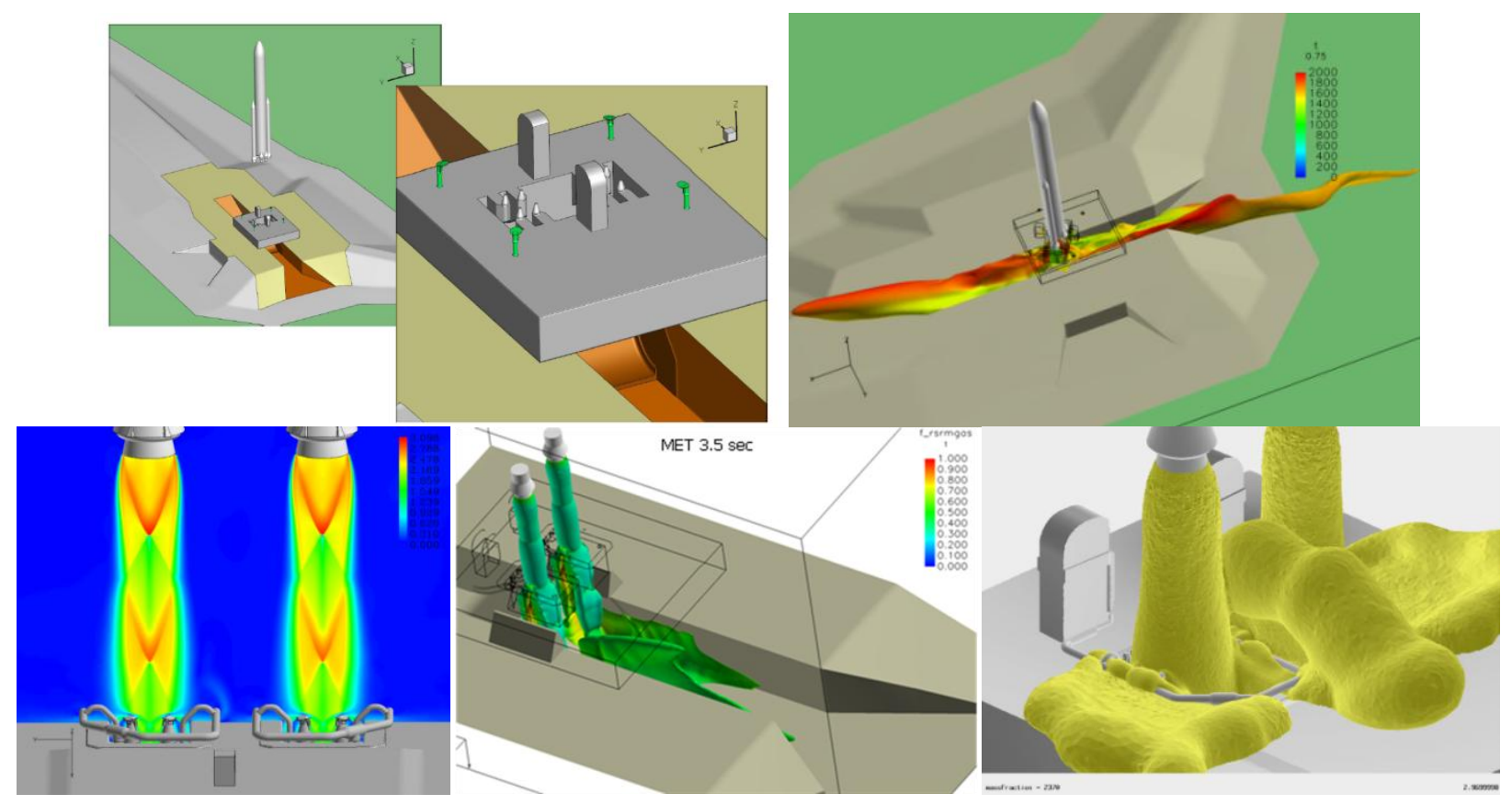

Figure 1: Examples of full launch pad CFD analysis

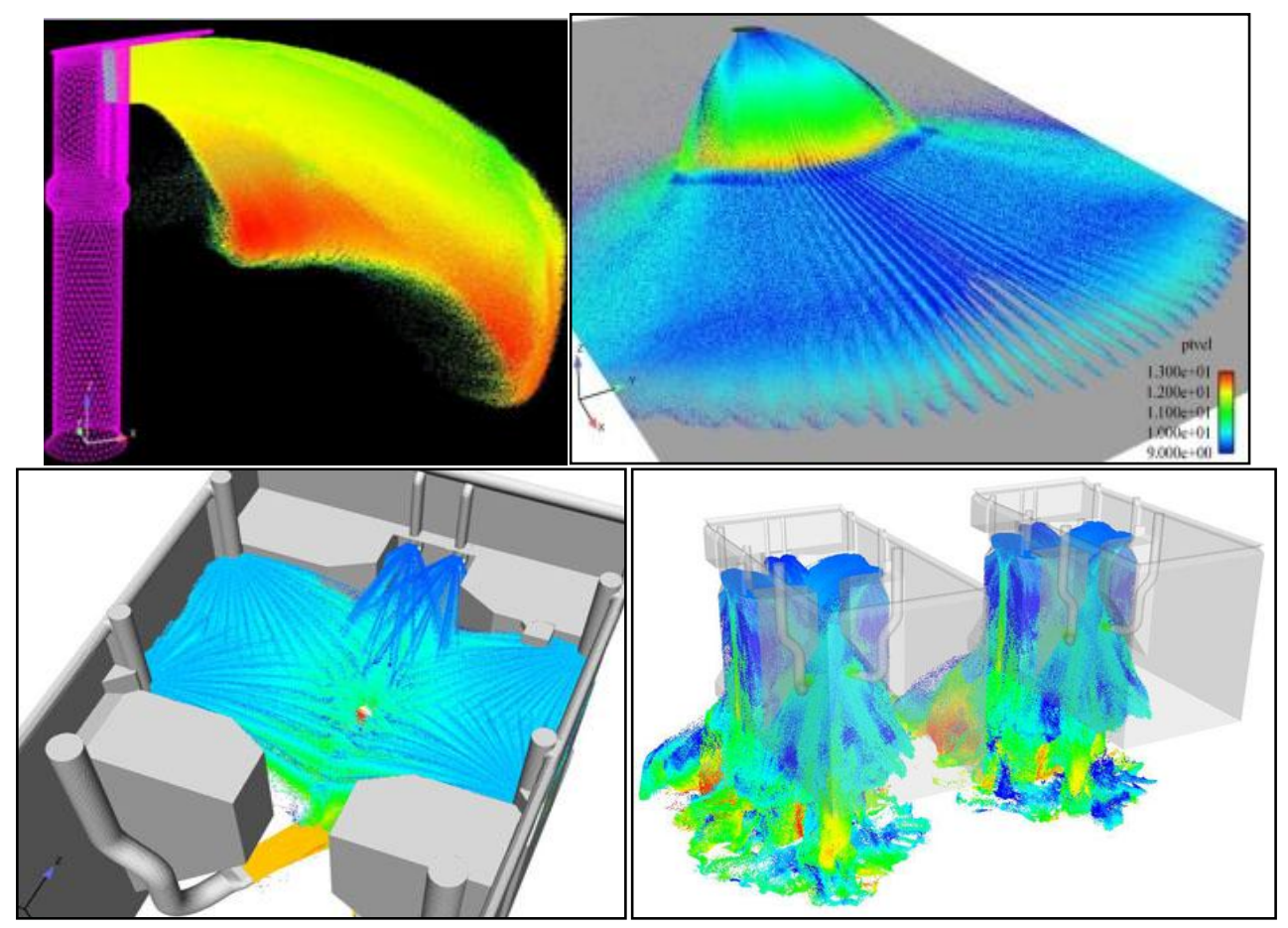

Figure 2: Multi-phase CFD modeling of deluge water injection on launch platform deck and in exhaust holes 


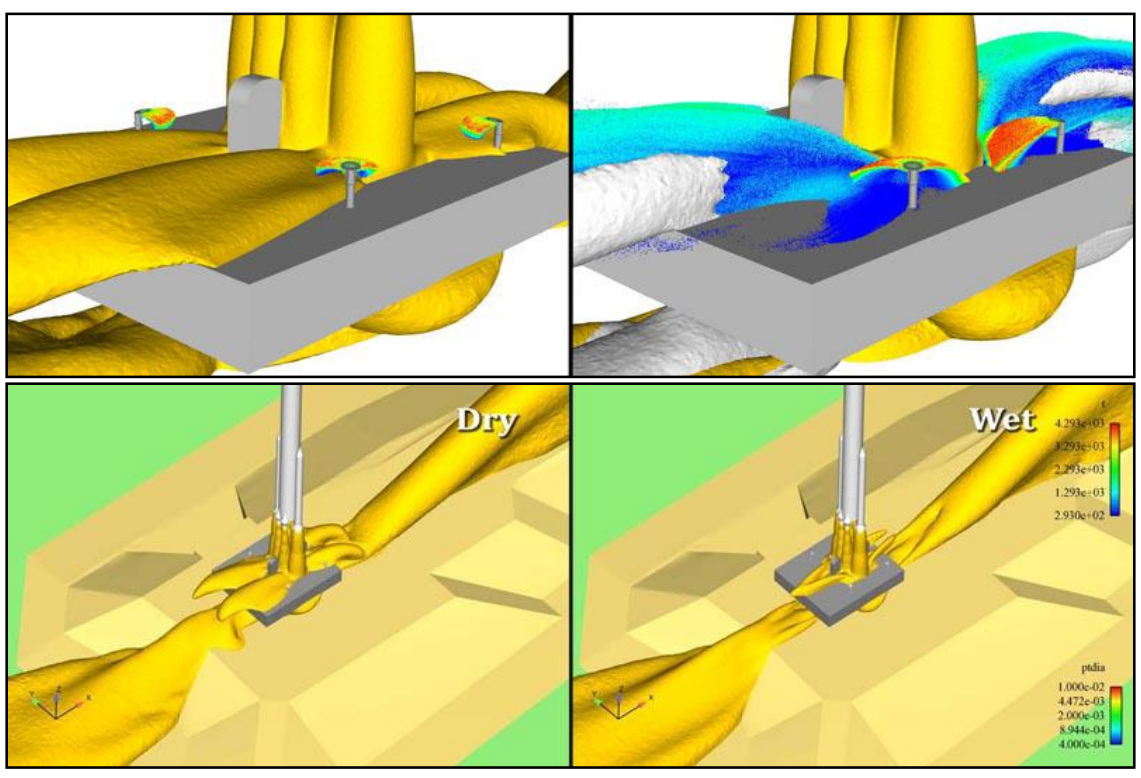

Figure 3: Effects of deluge water injection on launch pad plume characteristics

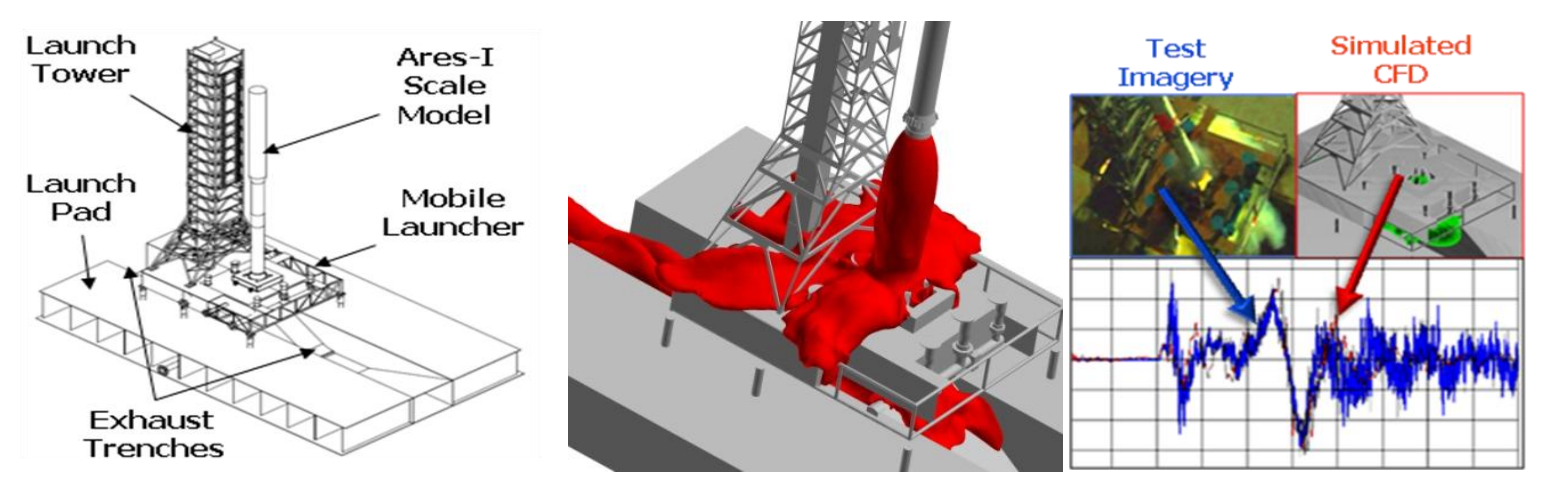

Figure 4: CFD Validation analysis against ASMAT scale test data

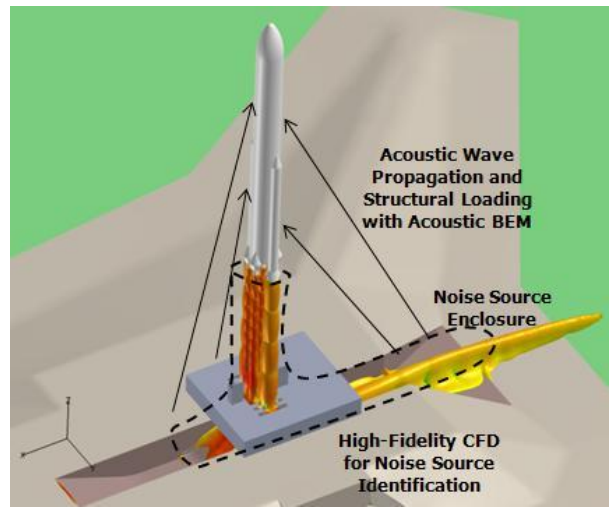

Figure 5: Concept of hybrid CFD-CAA modeling approach for farfield acoustic prediction 


\section{References}

1 Luke, E., and George, T., "Loci: A Rule-Based Framework for Parallel Multidisciplinary Simulation Synthesis, Journal of Functional Programming," Volume 15, Issue 03, 2005, pp. 477-502, Cambridge University Press.

2 Luke, E. A. ,"Loci: A rule-based framework for parallel multi-disciplinary simulation synthesis," Journal of Functional Programming, Special Issue on Functional Approaches to High-Performance Parallel Programming, 15(03):477-502. Cambridge University Press.

3 Luke, E., and Cinnella, P., "Numerical Simulations of Mixtures of Fluids Using Upwind Algorithms," Computers and Fluids, Volume 36, December 2007, pp. 1547-1566.

4 Counter D.D., Houston J.; "Ares I scale model acoustic test lift-off acoustics," J. Acoust Soc Am. 2011 Oct;130(4):2542.

5 Putnam G.C., Strutzenberg L.L.: "Simulation of acoustics for Ares I scale model acoustic tests," J. Acoust Soc Am. 2011 Oct;130(4):2543.

6 Liu, Y.J., Fast Multipole Boundary Element Method - Theory and Applications in Engineering, Cambridge University Press, Cambridge, 2009. 


\title{
Development of Modeling Capabilities for Launch Pad Acoustics and Ignition Transient Environment Prediction
}

\author{
Jeffrey S. West ${ }^{1}$ and Louise L. Strutzenberg ${ }^{2}$ \\ NASA Marshall Space Flight Center, Huntsville, Alabama, USA \\ Gabriel C. Putnam ${ }^{3}$ \\ All Points Logistics, Huntsville, Alabama, USA \\ Peter A. Liever ${ }^{4}$, and Brandon R. Williams ${ }^{5}$ \\ CFD Research Corporation, Huntsville, Alabama, USA
}

\begin{abstract}
$\underline{\text { Abstract }}$
This paper presents development efforts to establish modeling capabilities for launch vehicle liftoff acoustics and ignition transient environment predictions. Peak acoustic loads experienced by the launch vehicle occur during liftoff with strong interaction between the vehicle and the launch facility. Acoustic prediction engineering tools based on empirical models are of limited value in efforts to proactively design and optimize launch vehicles and launch facility configurations for liftoff acoustics. Modeling approaches are needed that capture the important details of the plume flow environment including the ignition transient, identify the noise generation sources, and allow assessment of the effects of launch pad geometric details and acoustic mitigation measures such as water injection. This paper presents a status of the CFD tools developed by the MSFC Fluid Dynamics Branch featuring advanced multi-physics modeling capabilities developed towards this goal. Validation and application examples are presented along with an overview of application in the prediction of liftoff environments and the design of targeted mitigation measures such as launch pad configuration and sound suppression water placement.
\end{abstract}

\section{Introduction}

The vibro-acoustic environment induced by acoustic energy released from a launch vehicle propulsion system during liftoff is of great concern for the integrity of the launch complex, the vehicle and payloads. Acoustic loads of $150 \mathrm{~dB}$ to $170 \mathrm{~dB}$ may reach sections of the launch vehicle, severely threatening the integrity of vehicle components or payloads. The launch vehicle noise originates from the supersonic rocket plumes, which convert a small portion of the propulsive energy to acoustic energy that radiates from the outer boundary of the plumes. Noise from supersonic rocket plume small scale turbulence radiates essentially omni-directionally while highly energetic Mach wave radiation has a strong aftward direction relative to the vehicle. The interaction and reflection of these highly energetic and directional waves from the launch facility structures back towards the vehicle are the main mechanisms for the high acoustic loads reaching the vehicle. Additional noise source regions are generated by plume spillage and impingement onto the launch pad deck as well as plume interaction in the flame trench.

Prediction and definition of the liftoff acoustic environment for NASA launch vehicles is the responsibility of the Fluid Dynamics Branch at the Marshall Space Flight Center. Engineering models based on empirical jet acoustic strength and directivity models (SP-8072) ${ }^{1,2}$ or correlations to scaled Saturn V measurements such as in the VAEPP (Vehicle Acoustic Environmental Prediction Program) ${ }^{3}$ are the current state-of-the-art production tools. These methodologies cannot account for design deviations from the original data used in the empirical models. An example is the effects of sound suppression water deluge effects which were not present in the original Saturn test

${ }^{1}$ AST Fluid Mechanics, Fluid Dynamics Branch/ER42.

${ }^{2}$ AST Fluid Mechanics, Fluid Dynamics Branch/ER42.

${ }^{3}$ Senior Engineer.

${ }^{4}$ Technical Fellow, AIAA Senior Member.

${ }^{5}$ Senior Engineer, AIAA Member.

American Institute of Aeronautics and Astronautics 
databases. Superimposing conservative empirical margin factors for these effects effectively blanketing all pad effects is typically applied to determine the bounds of design environments. These tools cannot be applied in proactively designing launch pad topology modifications or targeted placement of sound suppression water flow for maximum acoustic load mitigation effects. More detailed modeling approaches are needed that can model and identify the sources of noise generation and the effects of sound mitigation efforts. CFD simulation capability has matured to the point where it can be applied in this process.

Continuing improvements in CFD simulation capability and fidelity in the Fluid Dynamics Branch at MSFC have enabled CFD modeling of complete launch vehicles with multiple plumes interacting with detailed launch pad geometric models. These analyses have become invaluable in defining liftoff environments for Space Shuttle and the new Space Launch System (SLS) heavy lift launch vehicle. ${ }^{4}$ Time accurate simulations applying hybrid RANS/LES modeling are capable of capturing the sources of acoustic waves originating from the plumes and the plume interaction with the facility structures. Advanced multi-phase modeling capabilities such as the injection of deluge water in the CFD model have been added recently that enable the assessment of deluge water in affecting the acoustic source regions. These capabilities now offer the possibility of analysis support for the geometric design of the launch pad and targeted placement of water injection systems. The paper will present an overview of the technology components of the CFD analysis system and present selected validations against test data.

\section{Role and Requirements of CFD in Liftoff Acoustic Loads Prediction and Mitigation}

Launch vehicle acoustic loads can be subdivided into two distinct mechanisms: 1) Igniter pulse, ignition overpressure (IOP), and duct over-pressure (DOP) waves during startup of liquid engines and solid motors which produce low frequency, large amplitude, finite waves; and 2) noise generated by the supersonic plumes and their interaction with the launch platform and the flame trench that create high sound-pressure level loads all along the vehicle including the payload section.

Ignition over-pressure loads occur early during the launch phase while the vehicle is still on the pad (Figure 1). Mitigation measures include blockage of direct propagation paths to the vehicle by water troughs, essentially hammocks filled with liquid water, and injection of large amounts of water directly towards the plumes inside the launch platform openings. Simulation of overpressure waves with CFD require time-accurate simulations of motor nozzle start-up flow and propagation of the resulting mass and pressure wave fronts in the complex vehicle, launch platform, and flame trench geometry domain. The presence of the water injected has a profound effect on the wave propagation and must be included in the modeling.

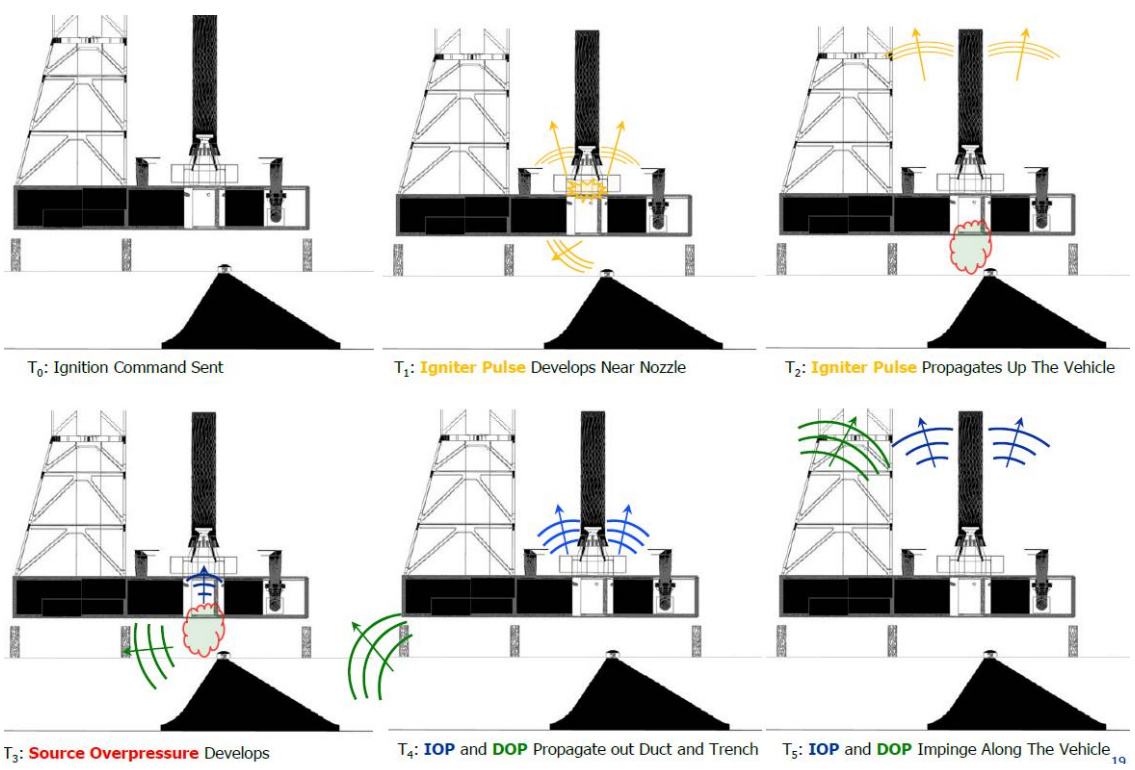

Figure 1: Sequence of solid rocket motor ignition transient environment.

American Institute of Aeronautics and Astronautics 
The rocket plume acoustic noise originates predominantly from the highly directional supersonic plume Mach wave radiation and subsequent reflection from the launch pad structures. Plume impingement on the launch platform as the vehicles ascends and the plumes spill and impinge on the deck generates new sources of noise, as does the plume impingement in the flame trench, noise propagation around the launch platform, and noise from the plumes exiting the flame trench. Peak plume noise occurs late in the liftoff phase, as the vehicle aft end rises to tower level and the plumes emerge from the launch pad flame holes. Mitigation measures consist primarily of injection of massive amounts of sound suppression deluge water onto the deck that suppresses the noise reflection and also dampens impingement noise sources.

CFD modeling must be capable of modeling the plume noise source mechanisms (plume mixing Mach waves) and plume impingement and spillage noise source regions across the frequency band of interest, which reaches beyond frequencies of $5000 \mathrm{~Hz}$. Properly resolved CFD simulations require time-accurate hybrid RANS/LES modeling for multiple plumes with diverse plume gas composition embedded in a detailed launch pad model. As deluge water injection is the main mitigation measure, CFD simulations must also be able to model the effects of the presence of water. Flow solver algorithms capable of performing simulations with flow physics of this complexity are typically second order accurate with considerable numerical dissipation for robustness. They are by nature tailored towards providing robust engineering analyses of complex propulsion flow field effects. Performing direct propagation of the acoustic waves originating from the resulting flow features requires considerably higher resolution and numerical accuracy.

It is not practical with today's CFD algorithms to achieve the required computational mesh resolution in a domain the size of a full size launch pad. With the SLS vehicle height of approximately $120 \mathrm{~m}$, the mobile launcher platform $30 \mathrm{~m}$ above the $200 \mathrm{~m}$ long flame trench, and the maximum liftoff acoustic loads occurring as the vehicle aft end is at tower height (approximately $150 \mathrm{~m}$ ), a computational domain of approximately $300 \mathrm{~m} \times 300 \mathrm{~m} \times 200 \mathrm{~m}$ is required. Current best practice for a second order accurate flow solver is to resolve acoustic wavelengths with 15 to 20 mesh cells. This results in cell sizes of approximately $0.15 \mathrm{~m}, 0.015 \mathrm{~m}$, and $0.003 \mathrm{~m}$ for frequencies of $100 \mathrm{~Hz}, 1000 \mathrm{~Hz}$, and $5000 \mathrm{~Hz}$, respectively. Mesh sizes on the order of $1 \mathrm{e}+9$ cells would be needed just for $100 \mathrm{~Hz}$, and $1 \mathrm{e}+14$ cells for resolving $5000 \mathrm{~Hz}$.

A hybrid approach of CFD and Computational Aero-Acoustics (CAA) appears to be the only viable approach for the foreseeable future to accurately propagate the noise to farfield locations such as the payload section at the top of the vehicle. However, this paper will demonstrate that the all-important capture of the noise source regions by performing full launch pad simulations with inclusion of sound suppression water effects is well within reach today. This capability allows the targeted implementation of mitigation measures directed at the noise source regions and also provides high-definition acoustic source location information to be passed on to the farfield load prediction models.

\section{Overview of Loci Framework and Loci/CHEM Flow Solver}

Loci/CHEM is the workhorse CFD analysis program used by the MSFC Fluid Dynamics Branch. The Loci framework ${ }^{5,6,7}$ was originally developed in the late 1990s with the goal of simplifying the development of complex numerical models that can take advantage of massively parallel high end computing systems. The framework provides a rule-based programming model whereby an application is described in terms of a collection of simple computational kernels. The Loci framework can assemble these kernels and optimize their scheduling on parallel high performance architectures. In addition, the framework is able to detect common programming errors by verifying that the algorithm conforms to a simple logical model. As a result, the Loci framework makes an excellent platform for the development and integration of a wide range of computational models. The framework supports the development of run-time loadable modules that allow Loci applications to be extended to support new physics and models with ease. Additionally, the verification capabilities of the Loci framework can provide assurances that the composition of these models satisfies rules of internal logical consistency.

The Loci/CHEM program ${ }^{3,8}$ was originally developed as a technology demonstrator for the Loci framework and has evolved into mature software for complex multi-physics simulations. The CHEM solver is a density-based NavierStokes solver employing high-resolution approximate Riemann solvers implemented for multi-component mixing and chemically reacting flows, and implicit time integration. In addition, the algorithms have been extended to

3

American Institute of Aeronautics and Astronautics

http://mc.manuscriptcentral.com/aiaa-maa12 
accurately model flows at low speeds through the use of preconditioning techniques. The program contains a variety of turbulence models including RANS, hybrid RANS/LES, and DES turbulence model treatments that include highspeed compressibility corrections. ${ }^{9}$

Loci/CHEM numerical models have been demonstrated to be at least second order accurate in space and time through rigorous verification using the Method of Manufactured Solutions (MMS). ${ }^{10}$ Loci/CHEM supports adaptive mesh refinement, ${ }^{11}$ simulations of complex equations-of-state including cryogenic fluids, ${ }^{12}$ multiphase simulations of dispersed liquid and solid particulates using both Lagrangian and Eulerian approaches, ${ }^{13}$ conjugate heat transfer through solids, ${ }^{14}$ fluid structure interaction modeling, ${ }^{15}$ and non-gray radiative heat transfer associated with both, gas and particulate phases. ${ }^{16}$

For complex geometry and object motion problems, the Loci/CHEM solver infrastructure supports overset meshes. Moving body simulations are performed with a 6-DOF model implementation capable of prescribed motion and unconstrained 6-DOF motion. The implementation supports simulations of multiple bodies, including bodies that have hierarchical relationships such as found when rotating components are part of larger systems (such as when propellers are included with the airframe). The dynamic overset mesh capabilities of Loci/CHEM have undergone extensive testing and validation studies ${ }^{17}$ and have been demonstrated to be accurate when compared with competing CFD methods. A rigid body contact and collision modeling capability is also under development to enable multiple-body proximate-flight simulation in the Loci/CHEM framework to simulate collision and recontact events for submunition dispensing, stage separation recontact, and debris object impact. ${ }^{18}$

Simulations of launch vehicle liftoff and staging events, which use several thousand computer nodes, are currently being executed using Loci/CHEM. In production mode, NASA has run simulations using Loci/CHEM with computational meshes reaching 500 million cells on more than 3,000 processors. ${ }^{19}$

The advances in CFD simulation capability and fidelity in the Fluid Dynamics Branch at MSFC have resulted in CFD modeling of complete launch vehicles moving on prescribed lift-off trajectories with multiple plumes interacting with detailed launch pad geometric models (Figure 2) ${ }^{20}$ These analyses have become invaluable in defining liftoff environments for Shuttle and future NASA heavy lift launch vehicle designs. Simulations with these tools are capable of capturing the sources of acoustic waves originating from the plumes and the plume interaction with the facility structures. Advanced multi-phase modeling capabilities such as the injection of deluge water in the CFD model have been added recently that enable the assessment of deluge water in affecting the acoustic source regions. These capabilities now offer the possibility of analysis support for the geometric design of the launch pad and targeted placement of water injection systems. 


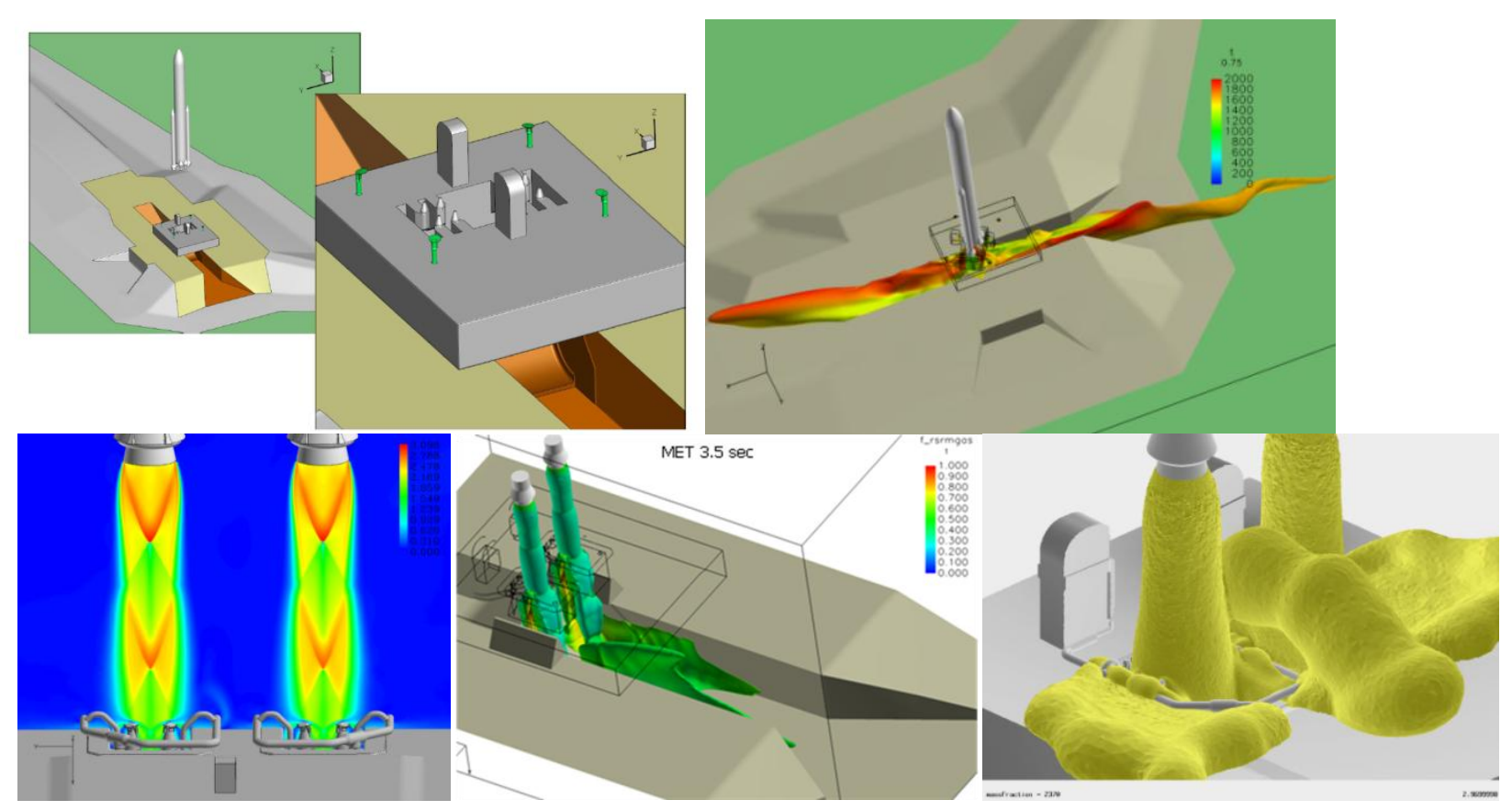

Figure 2: Examples of full launch pad steady, unsteady and moving vehicle CFD analyses with Loci/CHEM

Typical CFD simulations contain geometrical details that result in computational meshes with 150 to 200 million cells. Simulations are executed in time-accurate mode with hybrid RANS/LES turbulence modeling activated and time dependent rocket chamber start-up boundary conditions imposed. Wall clock time for IOP simulations covering approximately 0.3 seconds require supercomputer clock times of approximately 10 days and generate 2 terabytes of time accurate data for post-processing and animation of results. This makes simulations of this complexity and computational expense speedy enough to be applied in ongoing SLS vehicle and launch pad design analysis efforts.

\section{Validation of Liftoff Acoustic Field CFD Simulations}

A CFD model of the acoustic environments due to solid rocket motor ignition transients has been successfully validated, providing significant evidence that CFD models of this class are capable of capturing the sources of acoustic waves originating from the plumes and the plume interaction with the launch pad structures. The Ares I Scale Model Acoustics Test (ASMAT) was a series of live-fire tests of scaled rocket motors performed at the Marshall Space Flight Center. ${ }^{21}$ These tests were designed to simulate the acoustic environments of the Ares I launch configuration with a 5-percent scale model (Figure 3).
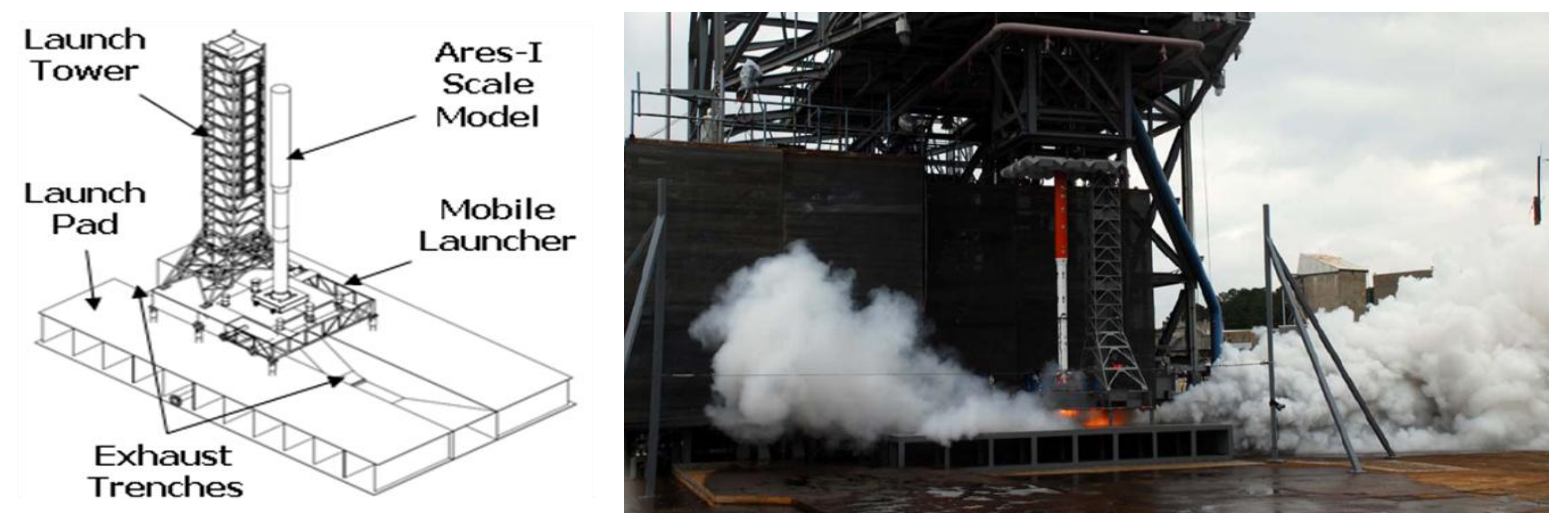

Figure 3: ASMAT 5\% scale model test

American Institute of Aeronautics and Astronautics 
The tests were heavily instrumented and provided a well documented set of high fidelity acoustic measurements useful for validation including data taken over a range of test conditions and containing phenomena like Ignition Over-Pressure and water suppression of acoustics. To take advantage of this data, a computational model of the ASMAT test setup has been constructed and test firings of the motor have been simulated using the Loci/CHEM computational fluid dynamics software (Figure 4). Results from ASMAT simulations with the rocket in both held down and elevated configurations, as well as with and without water suppression have been compared to acoustic data collected from similar live-fire tests. ${ }^{22}$

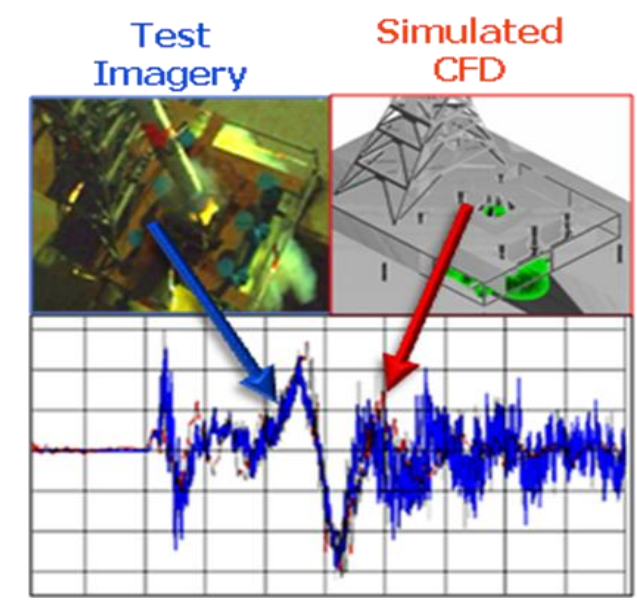

Figure 4: CFD Validation analysis against ASMAT scale test data

Figures 5 and 6 show the temporal pressure signal and the frequency content extracted at selected probe locations on the mobile launcher (ML) and pad surface, and on the vehicle surface far away from the plume. The pressure transient signal on the vehicle surface predicted by CFD compares very well with the actual test measurements (Figure 5). Major acoustic features have been well captured including the igniter pulse transient, and the sequence of ignition overpressure waves. The comparison of the frequency content at this location, however, shows that the CFD signal experiences significant dissipation for frequencies above $1000 \mathrm{~Hz}$. This is an indication of the dissipative nature of the CFD algorithms developed for robustness of the complex plume flow structure but with unacceptable dissipation and dispersion of the acoustic signal propagation. Figure 6 shows the comparison at probe points on the ML and pad surface located very close to the actual noise sources. The frequency content is well captured and preserved over the short propagation distance in the well resolved near-surface computational mesh. These findings confirm that the CFD program numerics are too diffuse for acoustic content tracking. A separate CAA approach is required for farfield acoustic field propagation. 

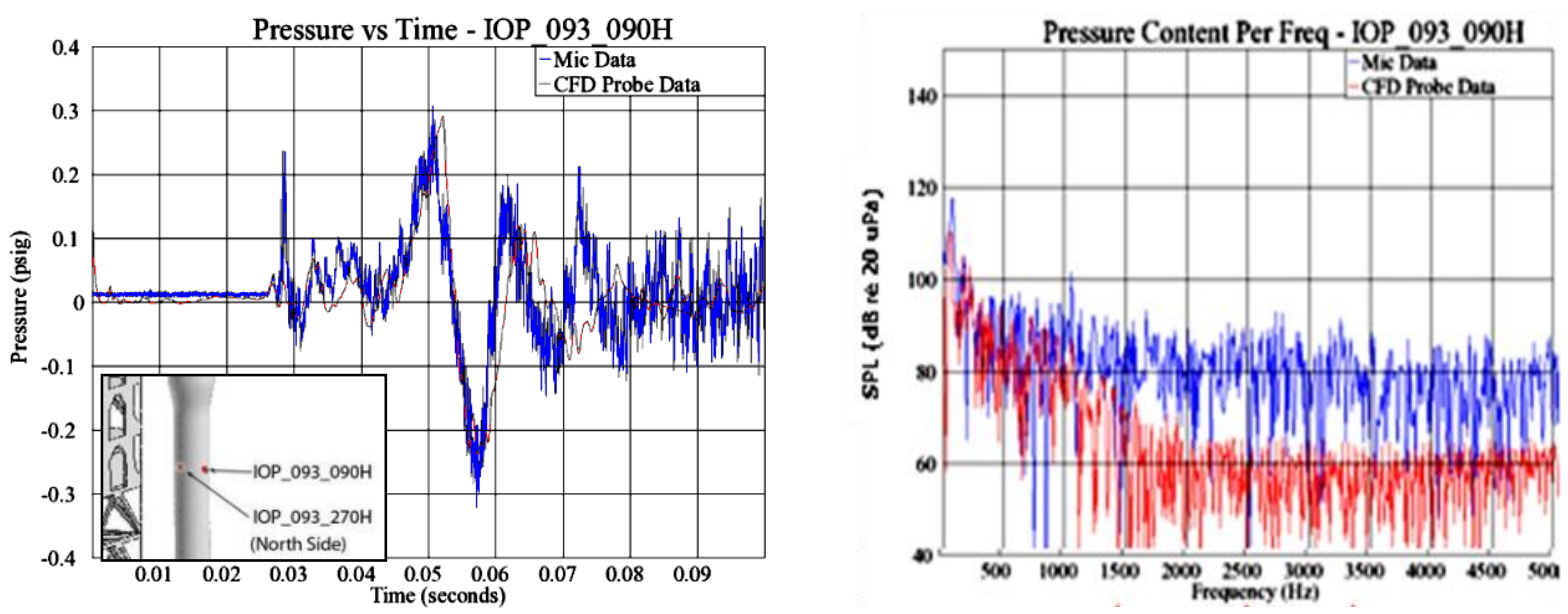

Figure 5: ASMAT CFD simulation: Temporal pressure amplitude (left), and frequency content (right) at probe location half way up vehicle.
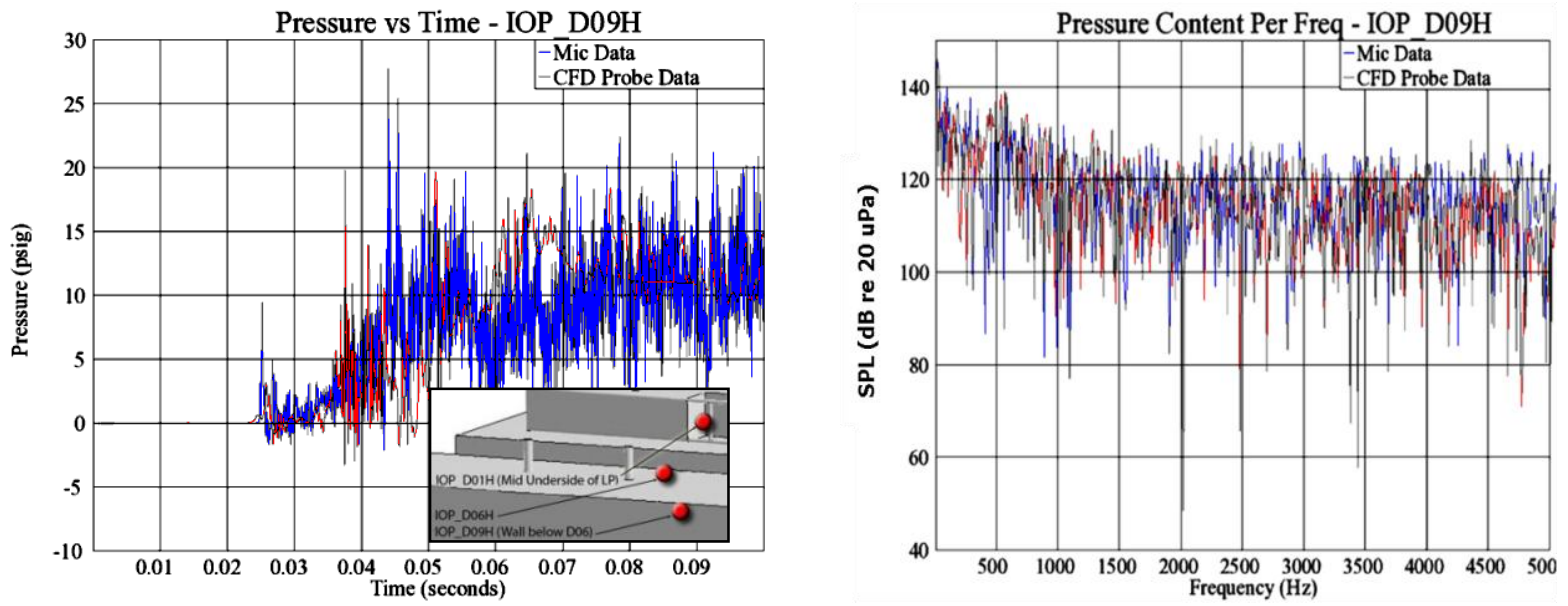

Figure 6: ASMAT CFD simulation: Temporal Pressure amplitude (left), and frequency content (right) at probe locations under mobile launcher and on pad surface.

\section{Identifying and Targeting Liftoff Acoustic Noise Sources}

A hybrid CFD/CAA application process involves several steps. The first step is to identify control surfaces enclosing the acoustic noise source regions to serve as input boundary conditions for the CAA process (Figure 7). All relevant sources of noise captured by CFD must be seamlessly enclosed by control surfaces to provide boundary conditions for the CAA computation. Unsteady flow characteristics extracted on these surfaces represent the nearfield core noise generation output and the acoustic field boundary conditions which are then propagated from this interface with appropriate acoustic field algorithms to characterize the farfield acoustic environment. 


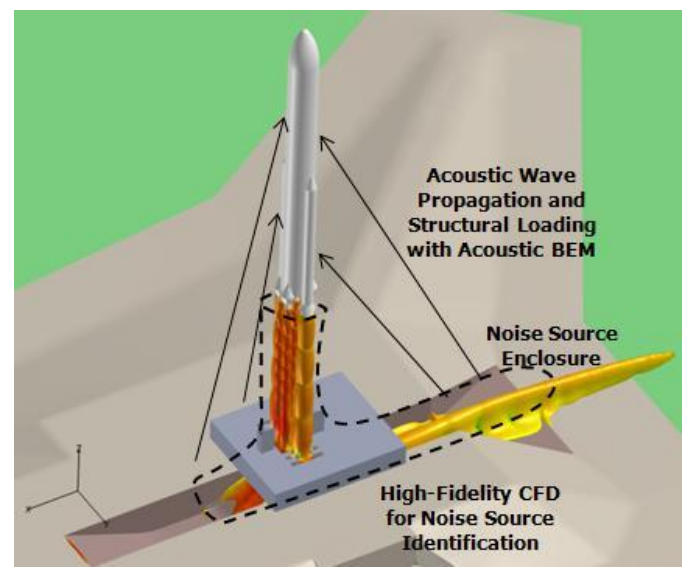

Figure 7: Concept of hybrid CFD-CAA modeling approach for farfield acoustic prediction

Enclosing surfaces resulting from plume interaction with launch pad structures are considerably more complex than for relatively simple free jet acoustics simulations. Both geometrical and flow physics complexity involved in the launch acoustic environment prediction complicates the process of identifying noise control surfaces with minimal user interaction. Various physical parameters have been considered in attempts to clearly identify the regions of noise sources in a CFD flowfield. Among them are turbulent kinetic energy, Reynolds stresses, velocity divergence, vorticity Q function, and others. In the current work, the most physically intuitive and insightful method of identifying noise source regions was obtained by visualizing the magnitude of the Lighthill stress tensor. ${ }^{23}$ Lighthill's equation, ${ }^{24}$ directly obtained from the governing laws of fluid dynamics, uniquely describes the physics of aerodynamic sound generation and propagation:

$$
\frac{\partial^{2} \rho^{\prime}}{\partial t^{2}}-c_{0}^{2} \frac{\partial^{2} \rho^{\prime}}{\partial x_{i}^{2}}=\frac{\partial^{2} T_{i j}}{\partial x_{i} \partial x_{j}}
$$

where $\rho^{\prime}$ is the density fluctuation field, $c_{0}$ is the speed of sound, and $T_{i j}$ is the Lighthill stress tensor that represents the sources of noise. The Lighthill stress tensor is commonly approximated as:

The Lighthill stress tensor has nine components:

$$
T_{i j} \approx \rho u_{i} u_{j}
$$

$$
T_{i j}=\rho u_{i} u_{j}=\left[\begin{array}{ccc}
T_{x x} & T_{x y} & T_{x z} \\
T_{y x} & T_{y y} & T_{y z} \\
T_{z x} & T_{z y} & T_{z z}
\end{array}\right]=\left[\begin{array}{ccc}
\rho u u & \rho u v & \rho u w \\
\rho v u & \rho v v & \rho v w \\
\rho w u & \rho w v & \rho w w
\end{array}\right]
$$

Considering only the dominant diagonal terms, one can write:

$$
T_{i j, d i a g}=\sqrt{T_{x x}^{2}+T_{y y}^{2}+T_{z z}^{2}}
$$

Using the magnitude of the $T_{i j, \text { diag }}$ approximation of the Lighthill stress tensor, distinct iso-surfaces can be obtained that represent only the noise generation region in a flow. Figure 8 shows the definition of the noise source regions for one of the ASMAT plume impingement simulations. The iso-surface of the Lighthill stress tensor magnitude clearly identifies the noise source regions. 

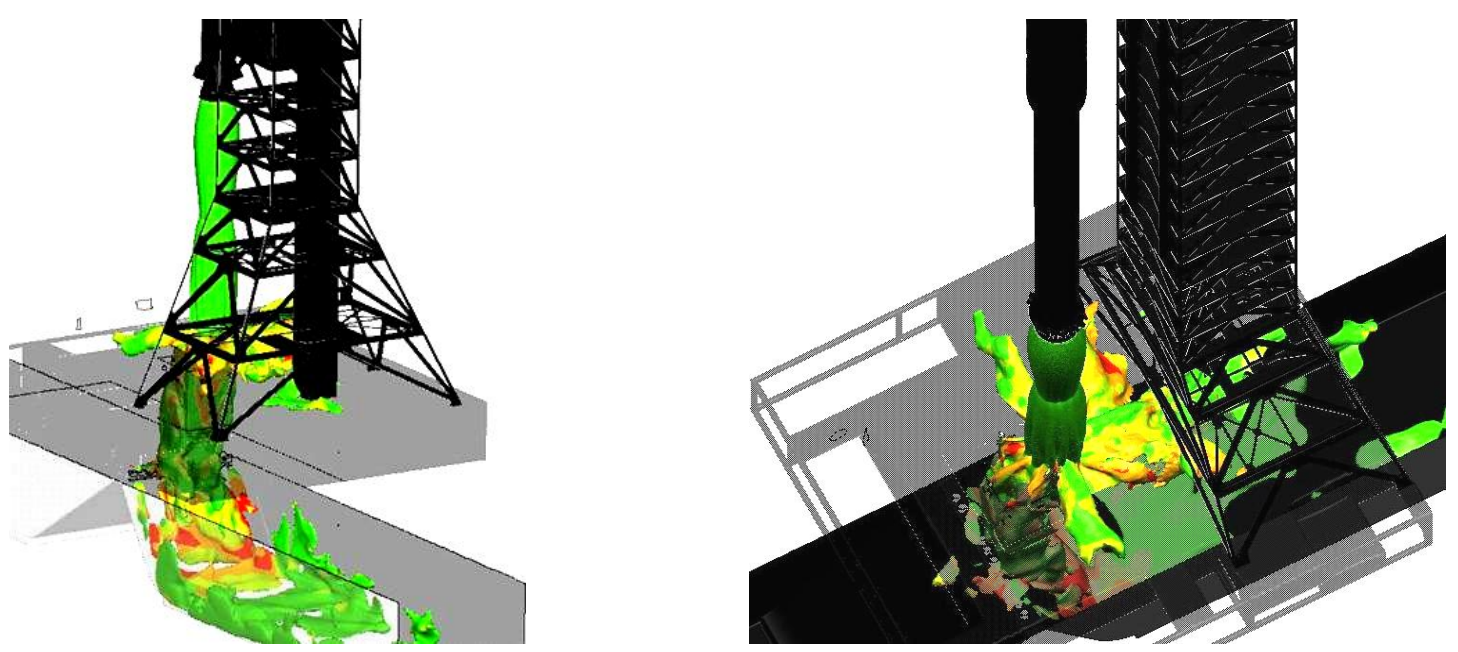

Figure 8. Visualization of Noise Sources for ASMAT Plume Impingement: Iso-surface of Lighthill Stress Tensor Magnitude $\left(T_{i, j, d i a g}=100,000\right)$

This noise source region information can now be used in two important ways for prediction and mitigation of liftoff acoustics. First, a geometrically simplified boundary surface can be defined that engulfs all acoustics sources near the pad. The initial condition inputs for a farfield acoustic field propagation method can then be extracted from the CFD simulation over this surface. This extraction allows for a bridge between CFD and CAA simulations and development of a coupled, hybrid CFD/CAA methodology which can quantify the acoustic loads on the full vehicle. Secondly, visualization of the extent and location of the noise source regions can be used for pinpointing mitigation measures using CFD. The effect of placing sound suppression water jets strategically aimed at noise source regions on the launch pad can be immediately visualized in changes in the Lighthill source regions. This enables CFD to become a targeted engineering design tool for sound suppression measures. Such measures include the placement and mass flow of sound suppression water jets as well as modifications to the launch pad structure geometry.

\section{Hybrid CFD-CAA Methodology for Liftoff Acoustic Load Prediction}

As demonstrated in the ASMAT validation examples, current CFD methodologies are numerically too dissipative and are not suitable to accurately capture the propagation of the acoustic waves from the plumes and the launch pad impingement regions to the top of the vehicle. A hybrid approach must therefore be applied that combines the highfidelity CFD analysis capable of identifying the acoustic sources with an efficient Boundary Element Method (BEM) that accurately propagates the acoustic field from the source locations. The BEM approach was chosen for its ability to properly account for reflections and diffraction of acoustic waves from structures such as the mobile launcher, flame trench and launch tower.

Initial efforts towards coupling of a BEM method for hybrid CFD/CAA have been performed through a NASA Small Business Technology Transfer project (STTR). ${ }^{25}$ The FastBEM acoustics software was applied to provide the BEM capability. ${ }^{26}$ FastBEM solves for propagation using the Helmholtz Equation via its Boundary Integral Equation (BIE) formulation. It uses a fast multi-pole (FMM) approach for solving 3-D, interior/exterior, radiation/scattering problems with velocity, pressure and impedance boundary conditions. Large-scale acoustic BEM models with up to several million unknowns or Degrees-of-Freedom (DOFs) have been solved on desktop workstations. Extension to larger models resulting from the large and complex topology of a launch vehicle and complete launch pad will likely require the parallelization and porting of the software to NASA supercomputer systems.

The process of extracting and passing the acoustic source strength information from the enclosure surface as input boundary conditions to farfield acoustic field propagation algorithms are being implemented utilizing existing Loci/CHEM native post-processing tools. 


\section{Integrating Sound Suppression Water Injection in Liftoff CFD Model}

For effective sound suppression, water must be injected into the plume environment as a dense, thick sheet of water. Subsequent interaction with the environment breaks the water up into progressively finer distributions of water particles. The water interacts with the plume environment in terms of mass, momentum and energy transfer. This interaction results in a reduction of the acoustic field generated by the plume environment as well as changes to the local mixture properties such as sound speed. The Fluid Dynamics Branch at NASA/MSFC has chosen the Lagrangian Particle Model available within Loci/CHEM to implement the application of water within the launch pad CFD simulations. This model has proven capable of modeling two-phase mixture effects of interest, among them the reduction of mixture sound speed important for launch pad IOP and acoustic wave propagation ${ }^{27}$.

Injecting massive amounts of water in the form of Lagrangian particles requires a CFD tracking of millions of water droplets. Simulations of launch pad water sound suppression system environments require flow rates of multiple 10,000 's of gallons per minute ( $\mathrm{gpm}$ ) from individual source locations, such as rainbird nozzles, and temporary peak flow rates of 900,000 gpm for the full launch pad water suppression system. Such vast increases in simulation scope relative to dry CFD models have the potential to reveal scalability issues with the CFD architecture which would not be encountered by unit problems, including inefficiency, resource utilization errors, and calculation errors due to particle number density. The ability of the Loci/CHEM Lagrangian two-phase model was tested for its ability of achieving full size high flow rates from single sources using a Space Shuttle rainbird ejecting water at tens of thousands gpm on a flat deck as shown in Figure 9. ${ }^{28}$ Testing has also expanded to simulations of the Solid Rocket Booster (SRB) water deluge system for the Space Shuttle, which ejects tens of thousands gpm of water from multiple opposing orifices in each SRB hole (Figure 10). ${ }^{29}$ This configuration served to test the ability to handle the large amounts of water particles in a geometrically extremely complex model. All test simulations were performed for the full scale launch pad size.
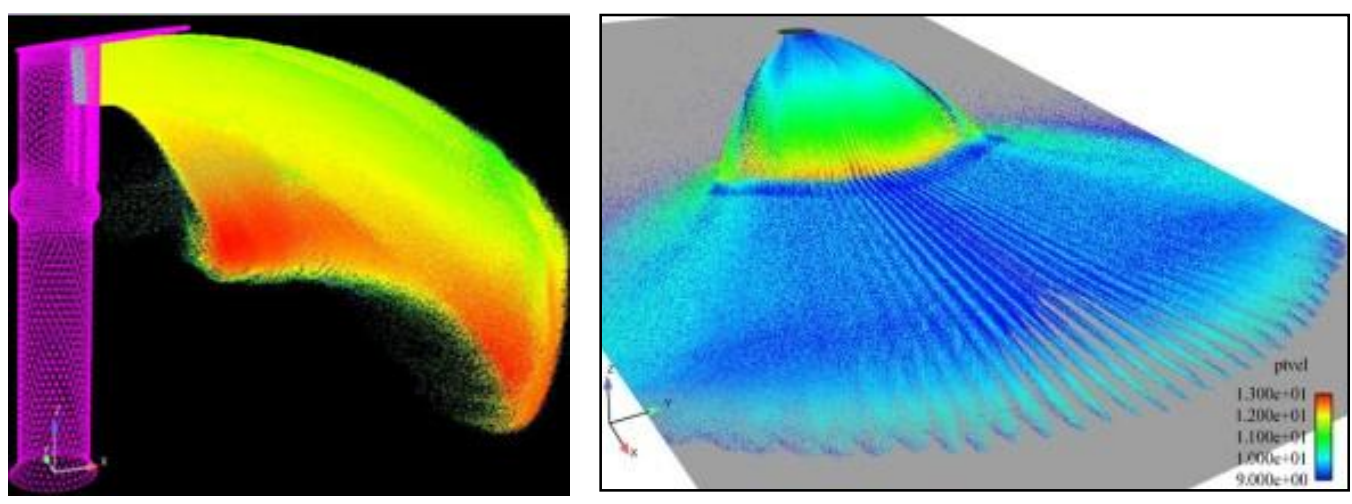

Figure 9: Multi-phase CFD modeling of deluge water injection on launch platform deck.
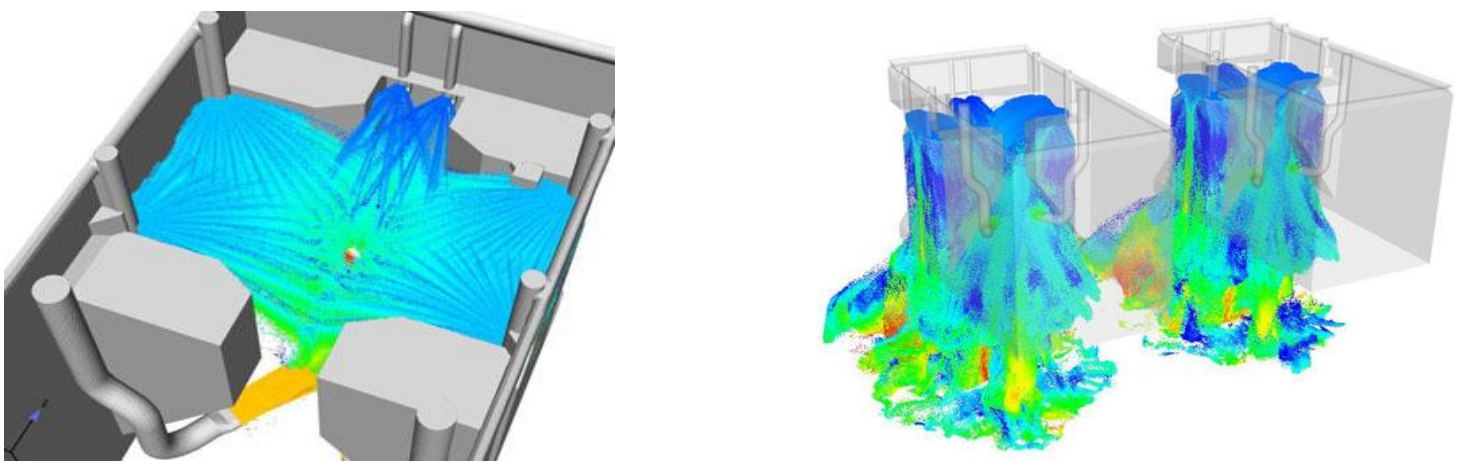

Figure 10: Multi-phase CFD modeling of deluge water injection in launch platform exhaust holes. 
The inherent parallelism of the Loci framework proved to be applicable with little deterioration for the extremely demanding multi-phase Lagrangian particle injection and phase change simulation required for sound suppression water effects modeling. These full-scale subsystem cases served to establish modeling best practices for the selection of suitable Lagrangian particle sizes for such massive water volume, proper parameters for particle breakup and phase transition modeling, and treatment of interaction with solid walls. While the model handles twoway exchange of mass, momentum and energy between the phases, important aspects of the injection process were simplified and must still be resolved in order to render a full fidelity CFD model. At present, the particles are not aware of neighboring particle presence and have no particle-particle interaction. This leads to unphysical amassing of water droplets in computational cells resulting it excessive local densities. Collisions, agglomeration and deflection of particles cannot be modeled at present. Secondly, when large amounts of particles hit the launch pad surface before they evaporate, the Lagrangian particle cloud must convert to a water layer on the surface. Approaches for solving this effect have been developed and are currently being pursued.

Using the Lagrangian module, an assessment of the Ares V launch environment acoustics has been performed which illustrates the effects of water suppression on acoustics ${ }^{30}$. To support this assessment, RANS CFD simulations of the full Ares V launch pad have been completed for both a dry configuration, which held the vehicle at a fixed height in space, and used no water systems, and a wet configuration, which had four on-deck rainbird systems activated and injected in excess of 100,000 gallons per minute of water in total. A side by side comparison of the plume environment characteristics is shown in Figure 11. After these simulations were completed, the turbulent kinetic energy on the mobile launch platform deck, which served as a surrogate measure of acoustic emissions, was compared for both cases to visualize the impact of rainbird systems on acoustic energy generation.

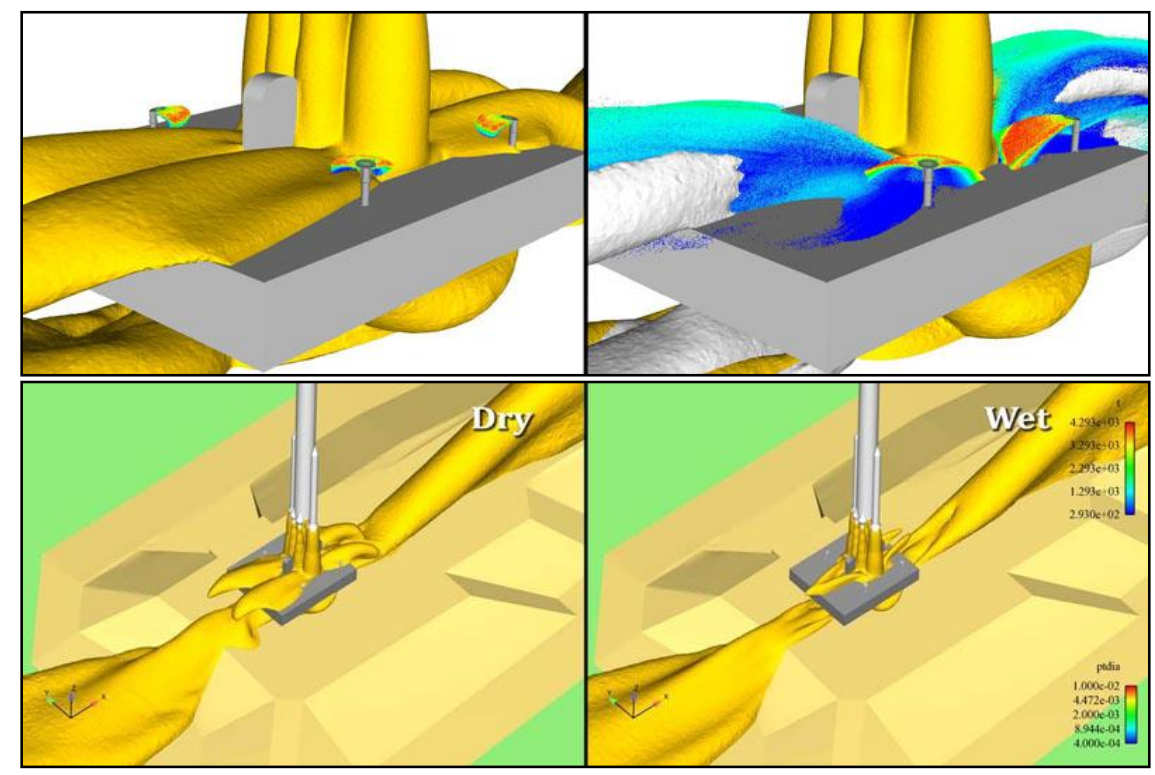

Figure 11: Effects of deluge water injection on launch pad plume characteristics

With the addition of the new methods for identifying acoustic sources presented in Section V, a systematic approach to identify regions of noise generation, target these regions for mitigation via geometrical changes or application of water and assess the effectiveness of the mitigation is now apparent. This as-yet to be validated computational mitigation assessment approach, coupled with limited experimental results, such as ASMAT results, provides the skeleton of a method to proactively design a launch pad for minimal acoustic environments,

\section{Ignition Over-Pressure Simulations in Support of SLS Launch Pad Design}

The development of the Space Launch System (SLS) vehicle and the supporting mobile launcher and launch pad structures requires characterization of the launch environment which includes the effects of the solid rocket booster 
(SRB) ignition overpressure (IOP). Existing IOP prediction methods developed for the Space Shuttle Program may not be capable of accurately modeling the impact on the SLS vehicle due to the much different engine placement and the return to a single mobile launcher flame hole for all engines.

CFD analyses have been performed of the IOP transient events for permutations of ML flame hole and main flame deflector in the flame trench together with vehicle configurations and positioning of the liquid engine and SRB nozzle exits to determine similarities and differences with the IOP environments experience with the Space Shuttle vehicle $^{31}$. These simulations are being used to provide guidance to the redesign efforts of the mobile launcher, the flame trench main deflector, and placement of sound suppression water. The CFD analyses were conducted using processes and procedures scaled from subscale validated predictions of the IOP environment for the ASMAT subscale tests conducted at NASA/MSFC completed in 2011. An assessment of the frequency-resolution capability of the full-scale CFD model compared to the sub-scale CFD model indicated that the full-scale model should be capable of accurate resolution of acoustic power contained in frequencies up to $150 \mathrm{~Hz}$ in the vicinity of the SLS vehicle base, main flame deflector and plume trench. ${ }^{32}$ This frequency is high enough to contain the engineering parameters of interest for IOP environment characterization.

Figure 12 shows an example of one early design cycle configuration. The placement of the SRB plumes close to the core engines and the impact and deflection of all plumes on one single side of the main flame deflector in this analysis results in potentially much stronger IOP impact on the vehicle. Figures 13 and 14 show three time occurrences of the highly energetic and acoustically complex pressure field in a plane through the SRB center along the flame trench and normal to the flame trench, respectively. Figure 15 shows the resulting impingement pattern and flow path of the core engines and SRB plumes in the flame trench and the impact pressure pattern on the flame deflector. The initial wave exiting the nozzle, the formation of the apparent IOP wave origin in the trench, and the propagation of the DOP down the flame trench are captured. Propagation of various waves through the ML flame hole and along the vehicle and waves propagation under and around the ML is also captured. This information on the path and strength of these waves reaching the vehicle provides valuable insight into the benefits or detriments of various permutations in the integrated vehicle, ML, and flame trench configuration.

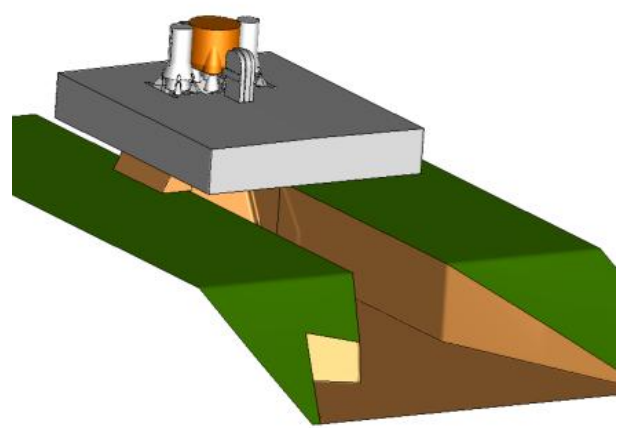

Figure 12. Example of SLS launch pad configuration CFD model
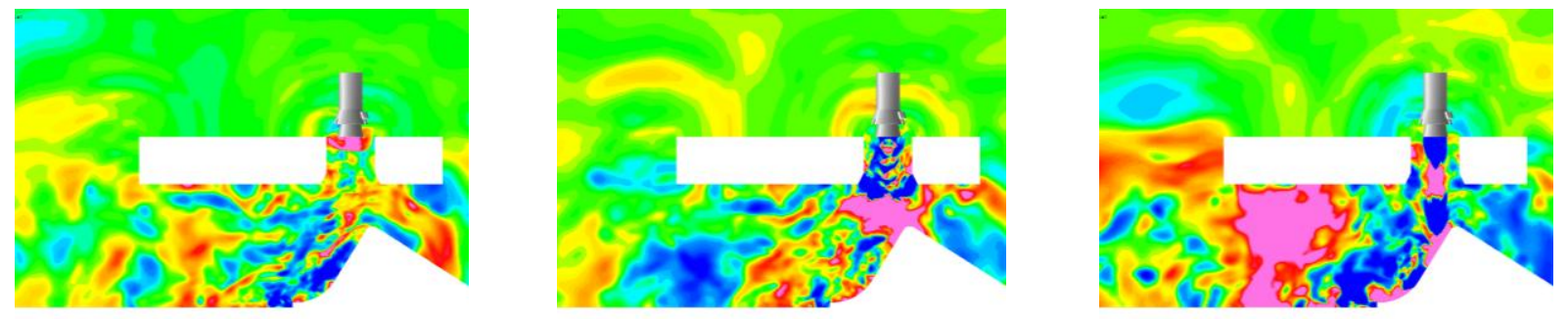

Figure13. Ignition overpressure field simulation for SLS launch pad in cutting plane through a single SRB 

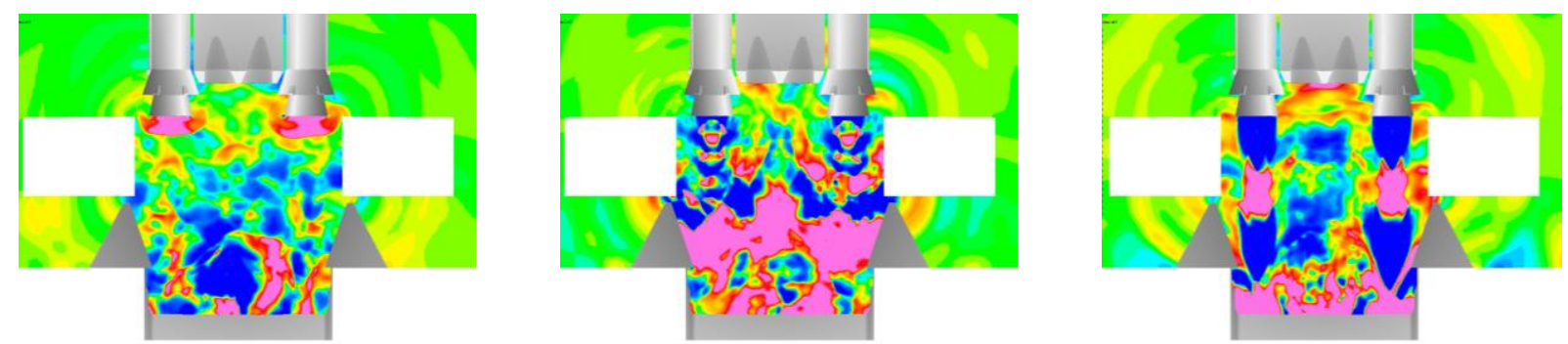

Figure 14. Ignition overpressure field simulation for SLS launch pad cross section plane through both SRBs
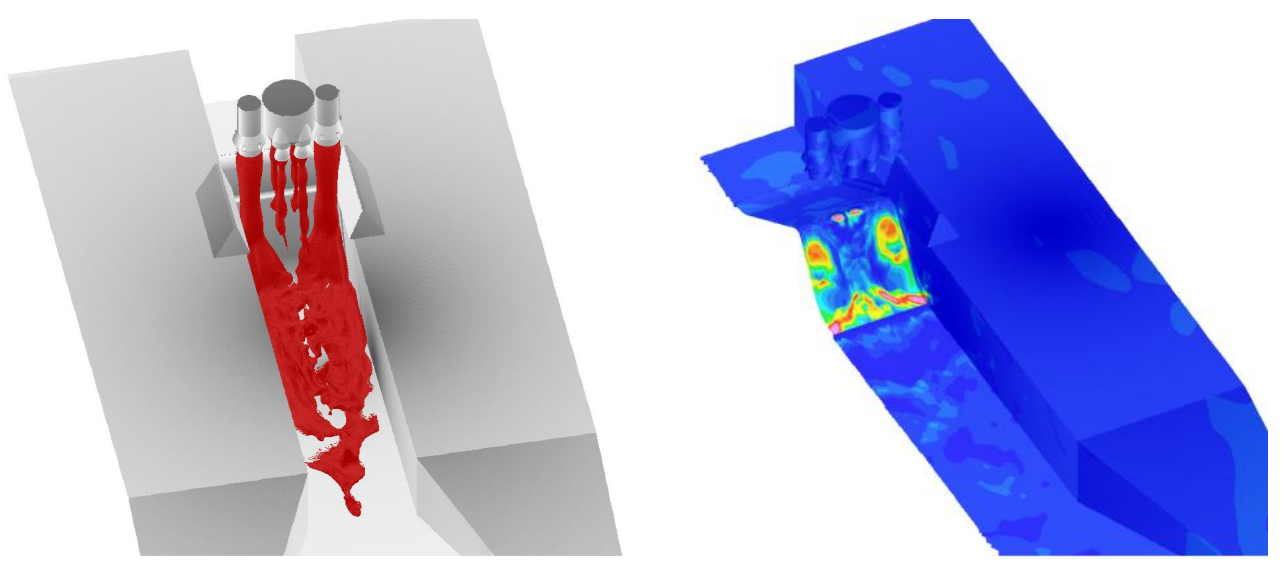

Figure 15. Ignition overpressure field simulation for SLS launch pad plume outer boundary (left) and flame deflector surface pressure (right)

\section{Future Plans}

Liftoff environment predictions through CFD simulations with the Loci/CHEM program are being used extensively in ongoing SLS design analysis cycles. Predictions and comparisons of ignition transient environments for the various layout permutations of the vehicle, the ML and flame trench will continue to provide early design guidance in the layout of the liftoff configuration for optimal acoustic load mitigation. In particular, the concept of identifying regions of noise sources in the CFD simulations and applying targeted placement of water injection aimed at those regions is being used as a potent design tool for designing sound suppression water systems.

Technology improvements that would increase the fidelity, accuracy and practicality of the current CFD tools have been identified and are being pursued. They include the implementation of higher accuracy CFD algorithms that are less dissipative and have lower numerical dispersion to allow accurate tracking of waves in the flowfield. The water sound suppression injection simulations with the current Lagrangian water particle injection models have shown high scalability and robustness. Improvements must be made in the particle interaction models to address particleparticle interaction and effects of agglomeration of water droplets resulting in surface pooling effects.

Efforts continue to establish a hybrid CFD-CAA methodology with acoustic field propagation by integrating coupling to a BEM program will fill the void of whole vehicle acoustic load predictions resulting from the inability of today's CFD numerics to propagate acoustic waves over large domains.

\section{Conclusion}

The advances in CFD simulation capability and fidelity in the Loci/CHEM framework used in the Fluid Dynamics Branch at MSFC have resulted in CFD modeling of complete launch vehicles with multiple plumes interacting with detailed launch pad geometric models. These analyses have become invaluable in defining liftoff environments for 
historical Space Shuttle and future SLS vehicle liftoff environments prediction. Simulations with these tools are capable of capturing the sources of acoustic waves originating from the plumes and the plume interaction with the facility structures. Advanced multi-phase modeling capabilities such as the injection of deluge water in the CFD model have been added recently that enable the assessment of deluge water in affecting the acoustic source regions. These capabilities now offer the possibility of analysis support for the geometric design of the launch pad and targeted placement of water injection systems. The ability of the Loci/CHEM simulation system to perform timeaccurate transient simulations with multi-engine motor transient boundary conditions for complex launch pad configurations in a timely fashion is now being applied to provide the definition of ignition overpressure environments for the SLS vehicle design and for the modification of mobile launcher, flame trench, and sound suppression water system configurations for minimizing liftoff environments.

\section{References}

1 Eldred, K., "Acoustic Loads Generated by the Propulsion System,” NASA SP-8072, June 1971.

2 Haynes, J., and Kenny, R. J., "Modifications to the NASA SP-8072 Distributed Source Method II for Ares I Lift-off Environment Predictions," AIAA Paper 2009-3160.

3 Counter, D. “Computer Program for Predicting Vehicle Acoustic Environment," ED33 (49-99), May 1988.

4 Strutzenberg, L.L. and Liever, P.A.: "Hybrid Computational Fluid Dynamics And Computational AeroAcoustic Modeling For Liftoff Acoustic Predictions," J. Acoust. Soc. Am., Oct 2011, 130(4):2543.

5 Luke, E. A., "Loci: A Deductive Framework For Graph-Based Algorithms," In Matsuoka, S., Oldehoeft, R., and Tholburn, M., Editors, Third International Symposium on Computing in Object-Oriented Parallel Environments, number 1732 in Lecture Notes in Computer Science, pages 142-153. Springer-Verlag, 1999.

6 Luke, E. A., "A Rule-Based Specification System for Computational Fluid Dynamics," PhD Thesis, Mississippi State University, Mississippi, 1999.

7 Luke, E. A., "Loci: A rule-based framework for parallel multi-disciplinary simulation synthesis," Journal of Functional Programming, Special Issue on Functional Approaches to High-Performance Parallel Programming, 15(03):477-502. Cambridge University Press.

$8 \quad$ Luke, E., "On robust and accurate arbitrary polytope CFD solvers," AIAA Paper 2007-3956.

9 Wilcox, D. C., Turbulence Modeling for CFD, DCW Industries, 2006.

10 Veluri, S., Roy, C. J., Luke, E., "Comprehensive Code Verification for an Unstructured Finite Volume CFD Code," AIAA Paper 2010-127.

11 Chalasani, S., Senguttuvan, V., Thompson, D., and Luke, E., "On the use of general elements in fluid dynamics simulations," Communications in Numerical Methods in Engineering, Vol. 24, No. 6, 2008, pp. 435-448.

12 Luke, E., and Cinnella, P., "Numerical Simulations of Mixtures of Fluids Using Upwind Algorithms," Computers and Fluids, Vol. 36, December, 2007, pp. 1547-1566.

13 Tong, X-L., and Luke, E., "Eulerian Simulations of Icing Collection Efficiency Using a Singularity Diffusion Model," AIAA Paper 2005-1246.

14 Liu, Q., Luke, E., and Cinnella, P., "Coupling Heat Transfer and Fluid Flow Solvers for Multi-Disciplinary Simulations," AIAA Journal of Thermophysics and Heat Transfer, Vol. 19, No. 4, 2005, pp 417-427.

15 Blades, E., Miskovish, S., Luke, E., Collins, E., and Kurkchubashe, A. (2011) "Multiphysics Simulation Capability using the SIMULIA Co-Simulation Engine," AIAA Paper 2011-3397,

16 Rani, S., and Luke, E., "Advanced Non-Gray Radiation Module in the Loci Framework for Combustion CFD," AIAA Paper 2008-5253.

17 Liever, P.A., "Validation of Loci/CHEM for predictions of shock impingement transient forces on a sphere," Technical Report ESTSG-FY09-00369, NASA MSFC, December 2008.

18 Harris, R.E., Liever, P.A., Luke, E., and Dudley, J., "Rigid Body Collision Modeling for Multiple-Body Proximate Flight Simulation in Loci/CHEM," 50th AIAA Aerospace Sciences Meeting, Nashville, TN, January 9-12, 2012, AIAA-2012-1236

19 Luke, E. A.. "Loci: Automated Synthesis for Terascale Computing Systems," 27th Army Science Conference, Orlando, Fl, November 29th - December 2nd, 2010, CP-08.

20 Radke, J. and Williams, B., "Space Shuttle: Time Accurate Computational Fluid Dynamic Simulation of a Pair of Moving Solid Rocket Boosters", Technical Report ESTSG-FY11-00146, NASA/MSFC, June 2011.

21 Counter, D, and Houston, J.; "Ares I scale model acoustic test lift-off acoustics," J. Acoust. Soc. Am., Oct 2011, 130(4), p. 2542. 
22 Putnam, G,C, and Strutzenberg, L, L,: "Simulation of acoustics for Ares I scale model acoustic tests," J. Acoust Soc. Am., Oct 2011, 130(4), p.2543.

23 Tosh, A., Owens, S. F., Sheta, S. and Habchi, S.D., and Liu, Y.J., "High-Fidelity Prediction of Launch Vehicle Liftoff Acoustic Fields," CFD Research Corporation, NASA STTR Phase I Final Report, Contract

NNX11CI36P, CFDRC Report: 9016-11/04, February 2012.

24 Lighthill, M. J., "On Sound Generated Aerodynamically: I. General Theory," Proc. of the Royal Society of London, Series A: Mathematical and Physical Sciences, 211(1107), 564-587, 1952.

25 Tosh, A., Owens, S. F., Sheta, S. and Habchi, S.D., and Liu, Y.J., "High-Fidelity Prediction of Launch Vehicle Liftoff Acoustic Fields," CFD Research Corporation, NASA STTR Phase I Final Report, Contract NNX11CI36P, CFDRC Report: 9016-11/04, February 2012.

26 Liu, Y.J., Fast Multipole Boundary Element Method - Theory and Applications in Engineering, Cambridge University Press, Cambridge, 2009.

27 Chamberlain, R. and Luke, E. A., "Multi-Phase Flow Analysis Tools for Solid Motor Applications", STTR Phase II Final Report, Contract NNX09CB73C, June 2011.

28 Putnam, G. "Simulations of Rainbird Water Spray with Lagrangian Particles Using Loci/CHEM", Technical Report ESTSG-FY10-02643, NASA/MSFC, September 2010.

29 Putnam, G., "Constellation: Simulation of Solid Rocket Booster Hole Water Deluge with Lagrangian Particles Using Loci/CHEM”, Technical Report ESTSG-FY10-02529, NASA/MSFC, September 2010.

30 Putnam, G., "Simulations of Ares V Launch Environment to Evaluate Impact of Water Suppression Systems", Technical Report ESTSG-FY11-00397, NASA/MSFC, January 2011.

31 Williams, B. "Analysis of Ignition Overpressure Simulations with Application to SLS", Technical Report ESTSG-FY12-465, NASA/MSFC, March 2012.

32 Williams, B. and Putnam, G. "Estimation of Full-Scale Space Launch System Simulation Accuracy Based on Ares Scale Model Simulation Validation”, Technical Report ESTSG-FY12-655, NASA/MSFC, May 2012. 


\section{Development of Modeling Capabilities for Launch Pad Acoustics and Ignition Transient Environment Prediction}

Jeffrey S. West and Louise L. Strutzenberg

NASA Marshall Space Flight Center, Huntsville, Alabama

\section{Gabriel C. Putnam}

All Points Logistics, Huntsville, Alabama

Peter A. Liever and Brandon R. Williams

CFD Research Corp., Huntsville, Alabama

$18^{\text {th }}$ AIAA/CEAS Aeroacoustics Conference

Colorado Springs, Colorado

June 4-6, 2012 


\section{Outline}

- NASA Liftoff Environment Analysis

- Traditional Engineering Methods and Important Role of CFD

- MSFC Liftoff CFD Capabilities

- Samples of Loci/CHEM capabilities

- Approach to Couple CFD with CAA for liftoff environment

- Identification of Liftoff Noise Source Regions

- Rationale for Selection of BEM based CAA

- Conclusions 
- MSFC Fluid Dynamics Branch responsible for NASA liftoff environments

- Standard approach for acoustic environment prediction: Apply empirical plume noise methods (VAEPP, SP-8072, ...)

- Resort to knock-down factors for effects not captured in empirical methods

- Launch pad topology uncertainties

- Sound reflections from complex pad geometry

- Plume impingement on launch platform

- Typically summed up as $+6 \mathrm{~dB}$ scale-up factor

- Water deluge sound suppression effects

- Would result in knock down factor

- Currently not used since no historical database available for credible bounds

- Mature, validated CFD capability in place for liftoff flow field analysis

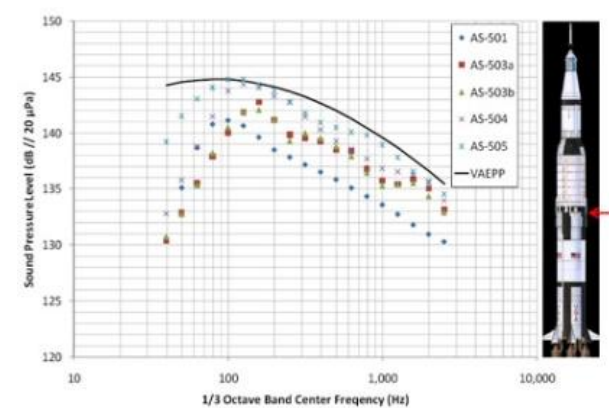

VAEPP

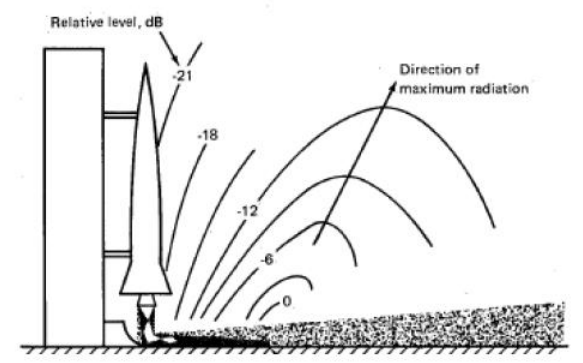

SP-8072

- Can we employ CFD to capture lift-off acoustic phenomena not captured by engineering methods? 


\section{MSFC CFD Analysis Activities}

- CFD analysis tool at MSFC is Loci/CHEM

- MSFC CFD analysis supports:

- Propulsion systems: Propellant delivery unsteady flows, combustion instability

- Liftoff plume induced environments: Mobile launcher, launch pad, and flame trench plume flow environments

- Ignition Over-Pressure (IOP) and start-up plume transient environment

- Plume and wind driven liftoff debris transport

- Launch pad hydrogen entrapment
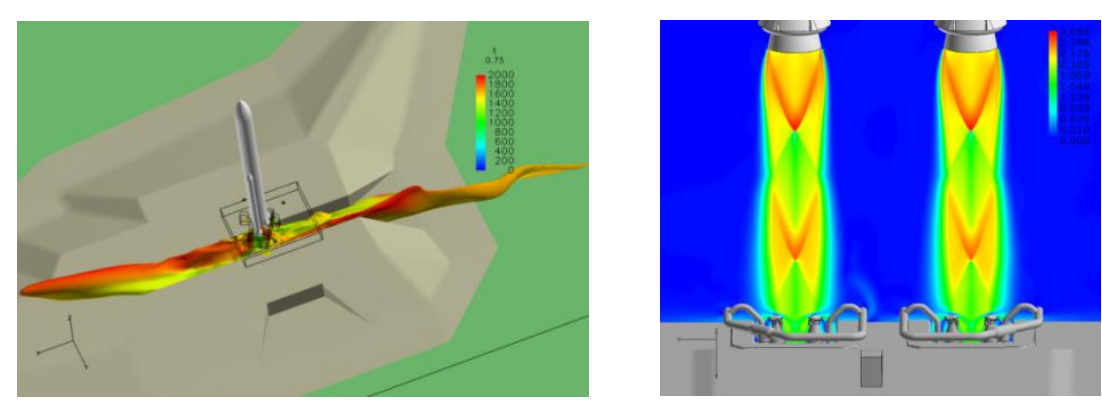

Launch Pad And Flame Trench Environment
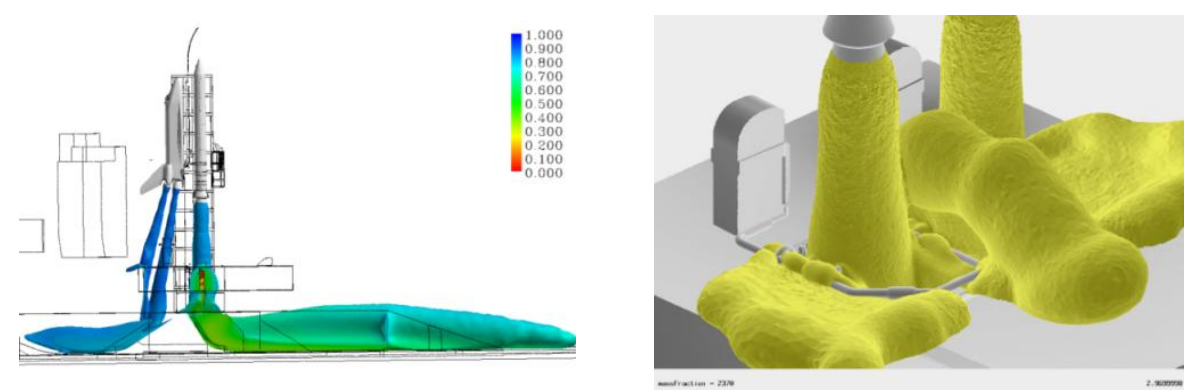

Space Shuttle, Ares, SLS liftoff plume flow
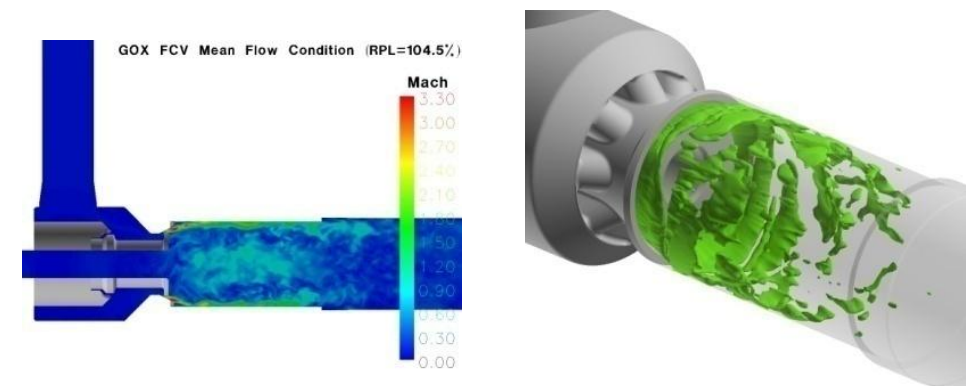

Hybrid RANS/LES Flow Control Valve $100 \mathrm{kHz}$ Acoustic Driven Fatigue Failure

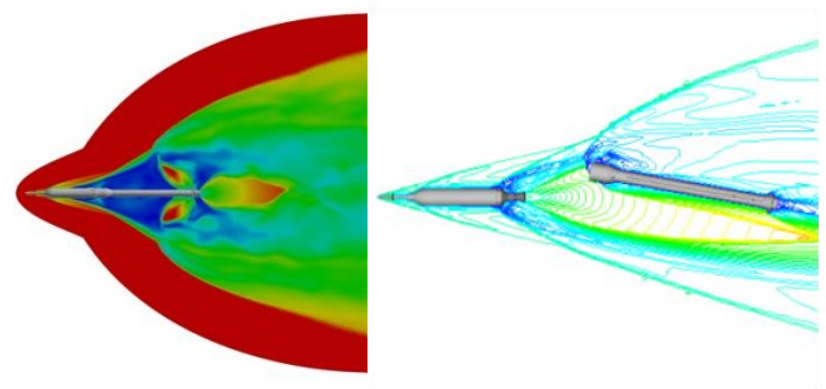

Ares I staging IOP

(Moving body + plume transient flow) 


\section{Loci/CHEM CFD Framework}

- Loci computational framework

- Highly scalable automatic parallelization platform for computational field simulations

- Developed at Mississippi State University by Dr. Ed Luke

- Open Source under the Lesser GNU Public License (LGPL) License.

- Loci/CHEM density-based Navier-Stokes solver implemented in the Loci framework

- Generalized unstructured grids

- RANS, URANS, DES, Hybrid RANS/LES turbulence modeling

- Eulerian multiphase models for particulates and droplets

- Lagrangian multiphase models for particulates and droplets with particle vaporization, condensation, combustion

- Real fluids EOS for cryogenic injection and combustion analysis

- Non-gray radiation transport models (particle and gas phase radiation)

- Solution adaptive mesh refinement with various error estimators available

- Mesh deformation for fluid-structure deformation and fuel burn-back surface

- Overset moving body with prescribed motion and 6-DOF

- Body Collision 6-DOF modeling

- Extensively verified using Method of Manufactured Solutions Technique

- $\quad 2^{\text {nd }}$ order space and $2^{\text {nd }}$ order time accurate, but not low dispersion/low dissipation

- Production simulations typically $10 \mathrm{M}$ to $300 \mathrm{M}$ cells on $3000+$ processors 


\section{Samples of MSFC CFD Capabilities}

- Acoustic driven loads

- SSME flow control valve fatigue fracture due to acoustic loading

- Engine start-up transients and Ignition Overpressure

- Ares-I Scale Model Acoustic Test (ASMAT)

- Water injection for launch pad water deluge simulation

- Two phase flow simulation with water droplet injection

- Effects of water phase on plume acoustic sources on pad

- Vehicle liftoff transient flow effects on pad and flame trench environment

- Full liftoff simulations staring from engine flow start-up transients all the way through liftoff trajectory vehicle motion

- Design support for Space Launch System (SLS) liftoff configuration

- Characterize ignition overpressure and flame trench plume impingement environment

- Provide input to mitigation measures design (launch pad geometry, sound suppression water injection, ...) 


\section{CFD for Internal Acoustics}

- Application in capturing internal flow acoustics

- Space Shuttle fuel flow control valve supersonic flow and impingement

- Exciting tangential and radial mode cavity acoustics

- Valve poppet developed crack due to high cycle fatigue

- Loci/CHEM Hybrid RANS/LES simulations

- Captured occurrence of various tangential and radial modes up to $100 \mathrm{kHz}$

- Excellent correlation with FEM modal analysis and failure mode forensic analysis

- Validated against calibration rig tests
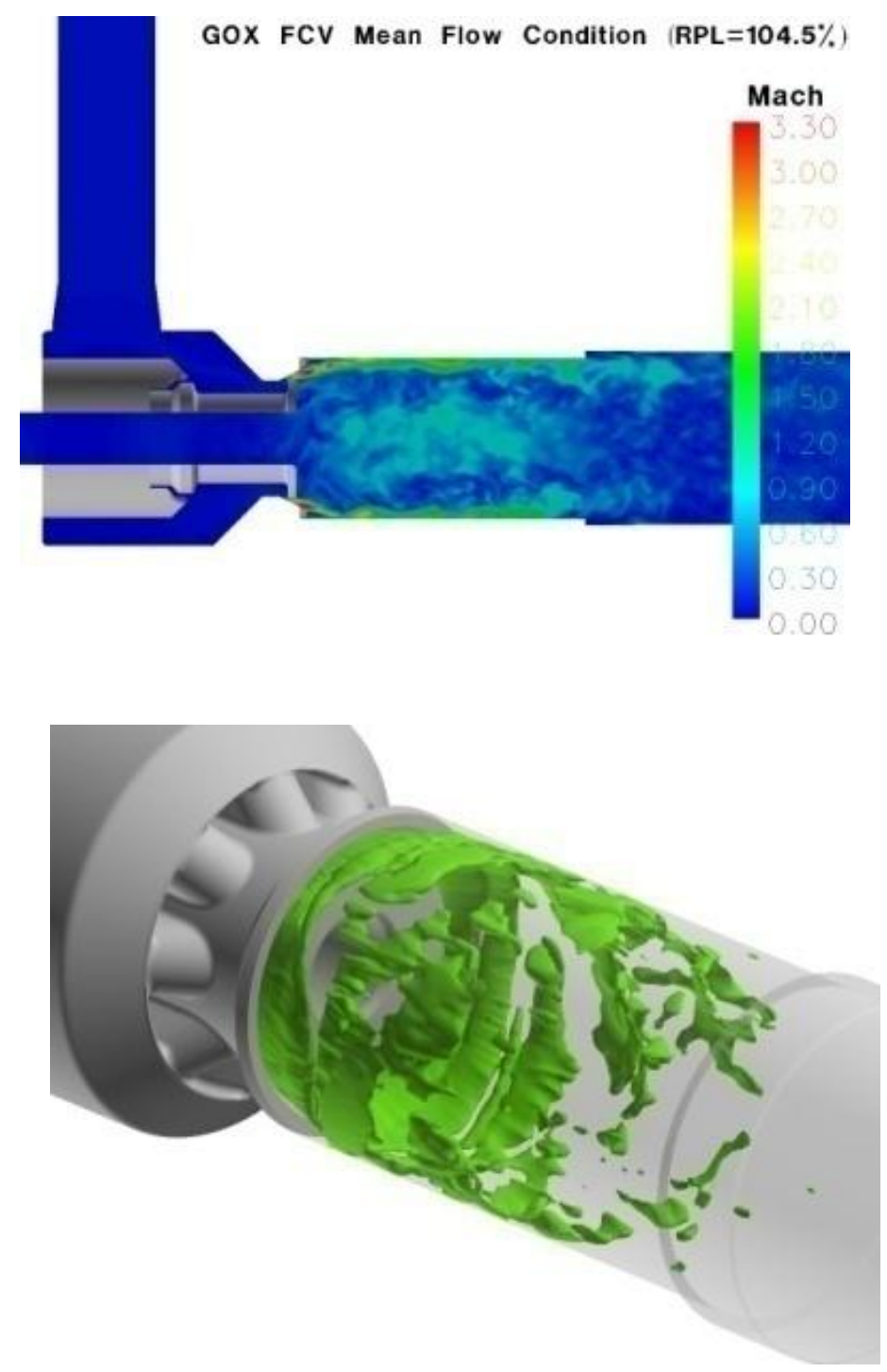


\section{ASMAT IOP Simulation}

- $\quad$ Ares-I Scale Model Acoustic Test (ASMAT)

- Tests of $5 \%$ scale model of Ares I vehicle

- Scale model powered by Rocket Assisted Take-Off (RATO) motor

- Stationary during firing

- 100+ pressure transducers on launch structure and vehicle

- Tests performed with and without water deluge

- Valuable Source for CFD validation

- Demonstration of CFD capability for IOP prediction

- Well documented set of high fidelity measurements suitable for CFD validation
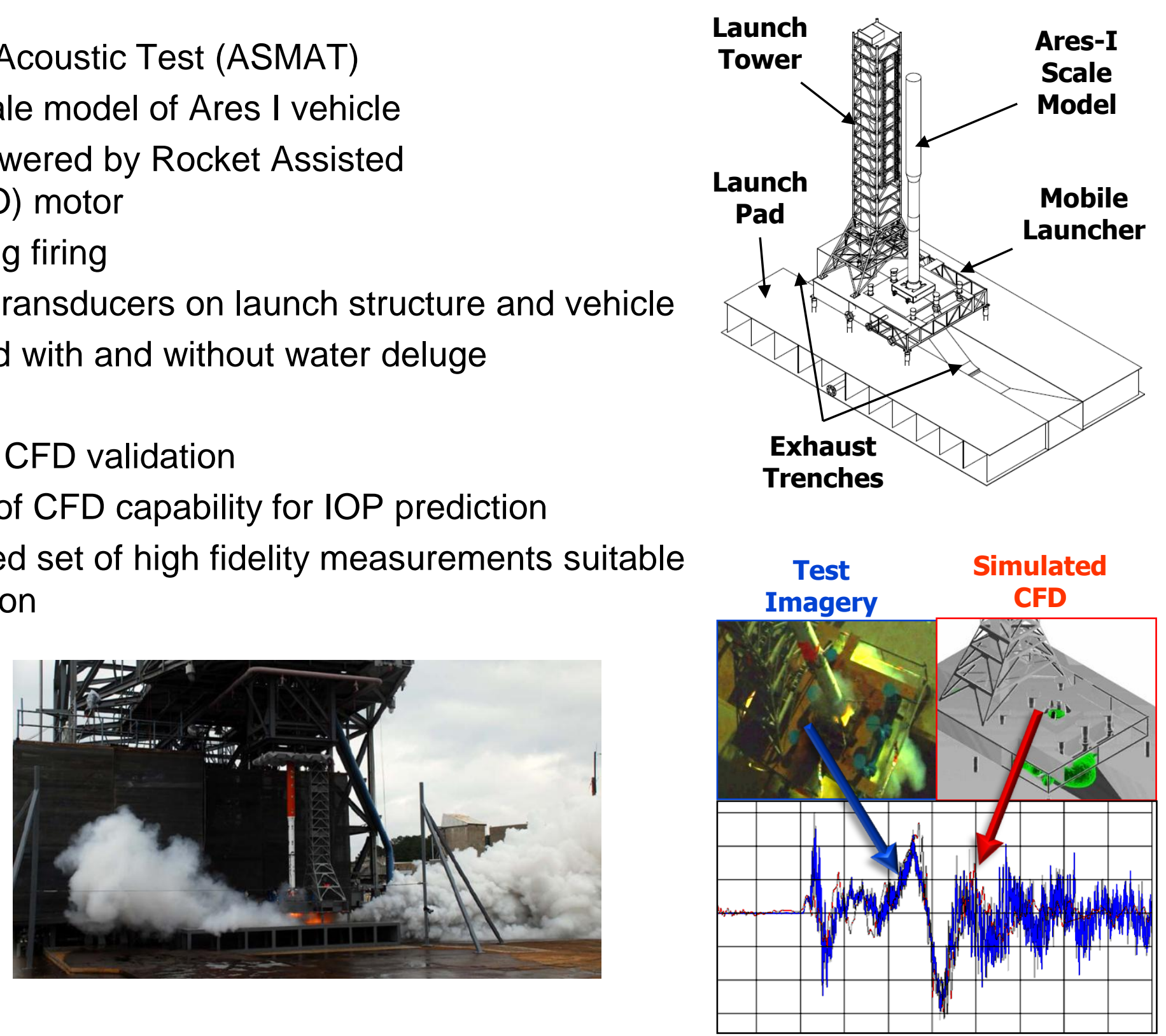
- Quantitative comparison of time and freq domain signals

- Major pressure peak amplitudes and timings captured with 5-10\% error

- Nearfield (sensor close to source) frequency content captured well

- Farfield frequency content lost above $\sim 1000 \mathrm{~Hz}$

- Launch vehicle acoustic frequencies must be resolved to $5 \mathrm{kHz}$ minimum

- Requires improved grid resolution and algorithms for acoustic content tracking or separate CAA acoustic field propagation approach
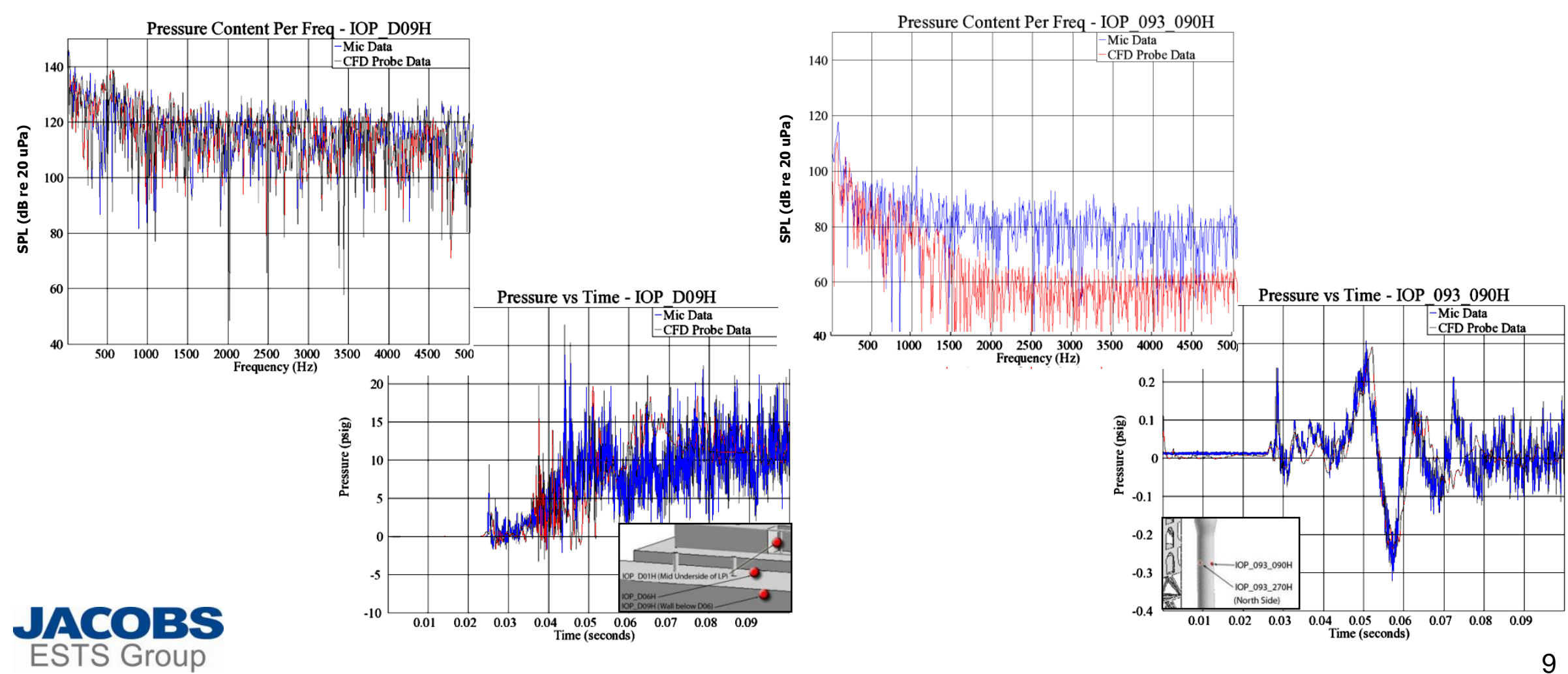


\section{Launch Pad Water Deluge Effects}

- Pathfinder simulations of water injection into launch pad plume environment for SLS concepts

- LC39 launch pad with detailed flame trench

- Launch pad with rainbirds emitting sprays of water

- Mixing of liquid engine and SRB plume composite gas mixtures

- Lagrangian particle model in Loci/CHEM used to model the water spray with water drop break-up and phase change

- Approx. 200M cells, Tracking Tens of millions of particles
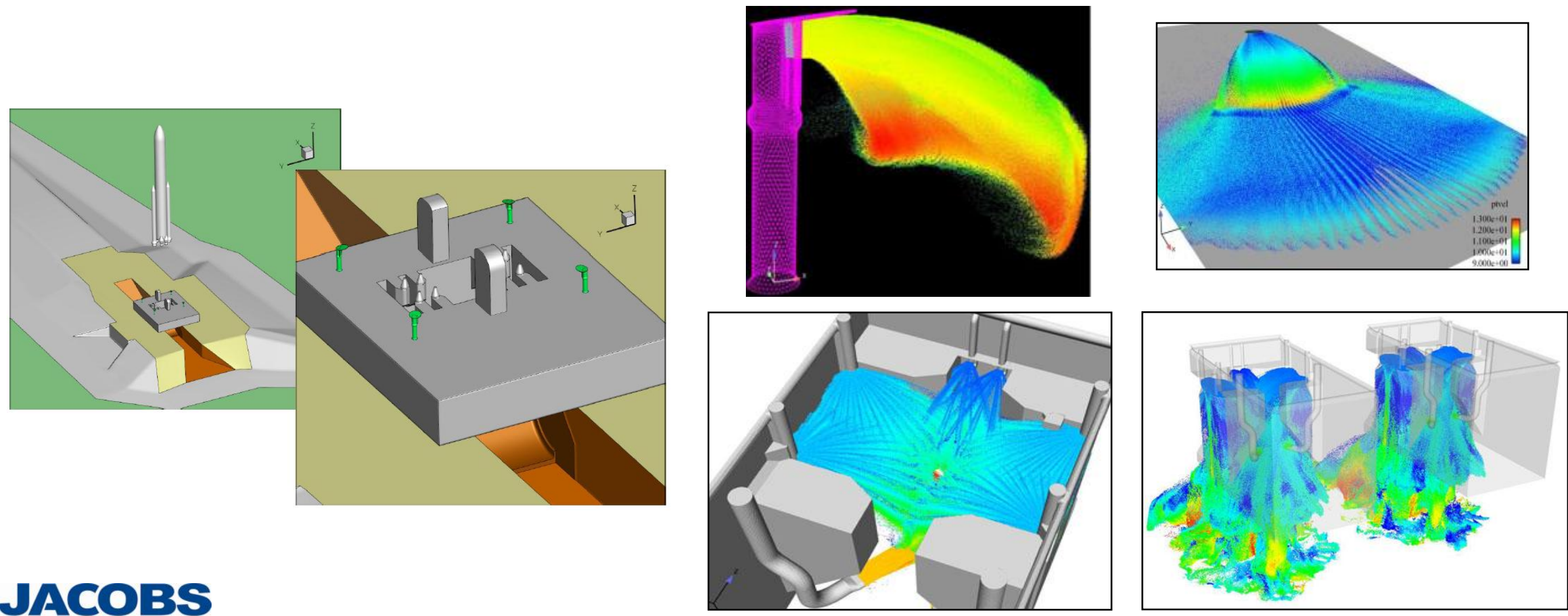


\section{Launch Pad Water Deluge Effects}

- Presence of water on deck surface considerably changes turbulent kinetic energy regions from plume spillage

- Alters impedance for plume Mach wave noise reflection

- Pathfinder CFD simulations demonstrated possibility of multi-species, multi-plume liftoff simulations with multi-phase gas-water effects

- Practical design application: Use CFD modeling to target regions of high turbulent kinetic energy to reduce noise sources

- Support launch pad design with targeted placement of rain birds for maximum acoustic mitigation effect

- Challenge will be to resolve acoustic wave propagation in direct simulation: hybrid CFD-CAA seems only reasonable approach
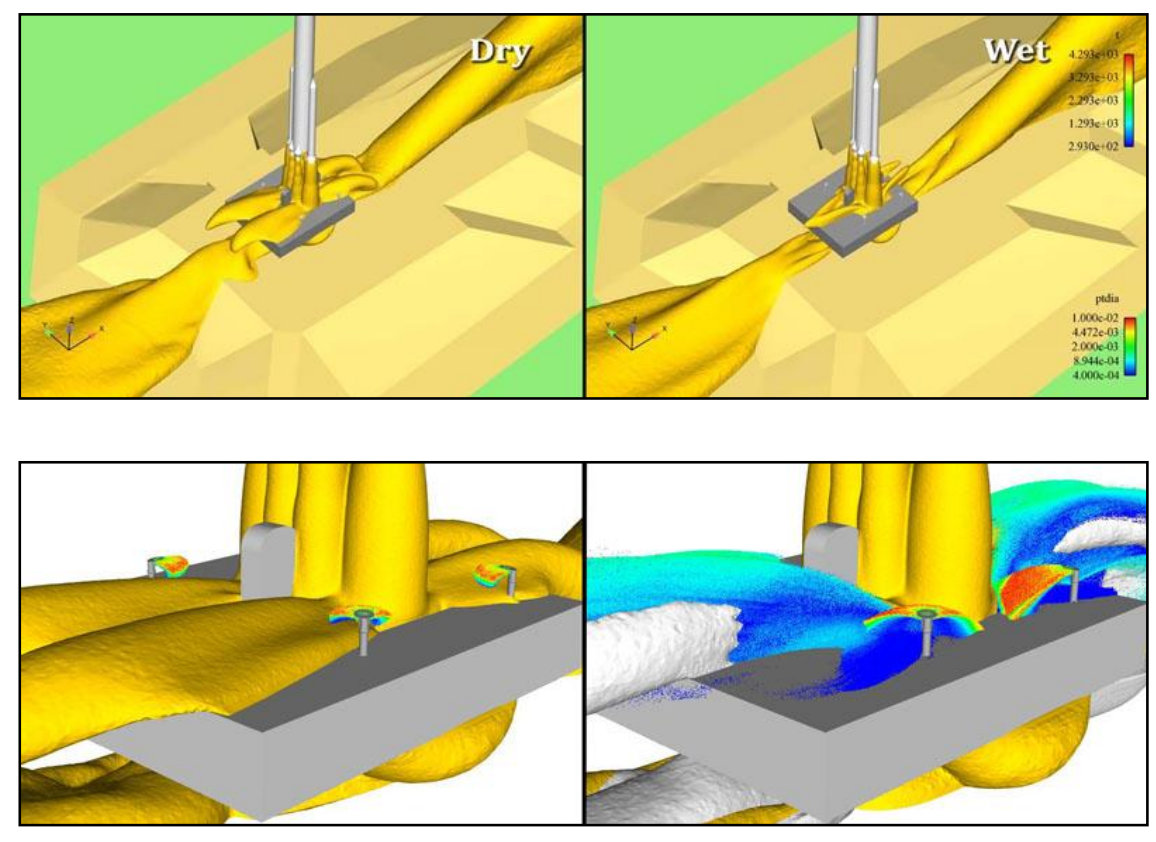

Reduction of Plume $500 \mathrm{~K}$ Iso-Surface from 0.7 to 4.5 seconds

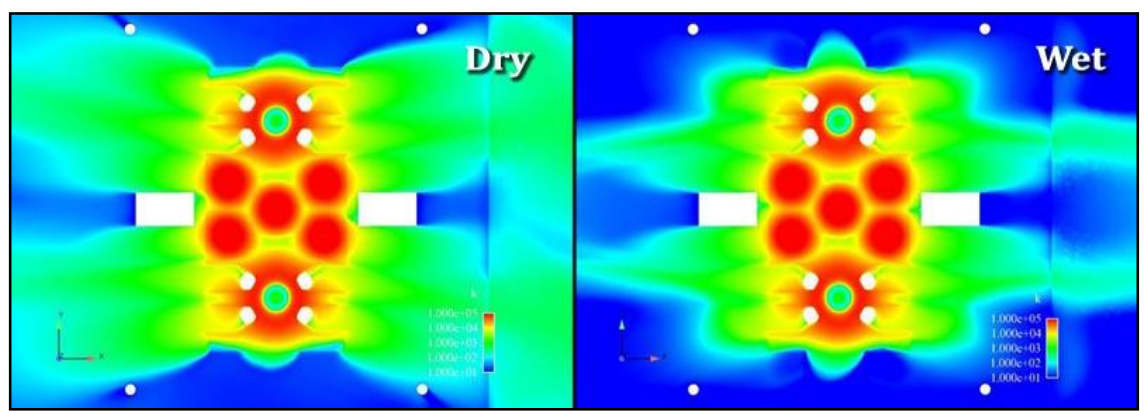

Reduction of turbulent kinetic energy at deck level 


\section{Shuttle Liftoff Plume Flow}

- Space Shuttle SRB plume impingement environment

- Transient trench flow starting from SRB ignition followed by moving body plume transient plume impingement environment

- Captures transient start-up flow and IOP under launch pad and plume spillage onto pad deck during liftoff motion

- Overset grid 6-DoF simulation
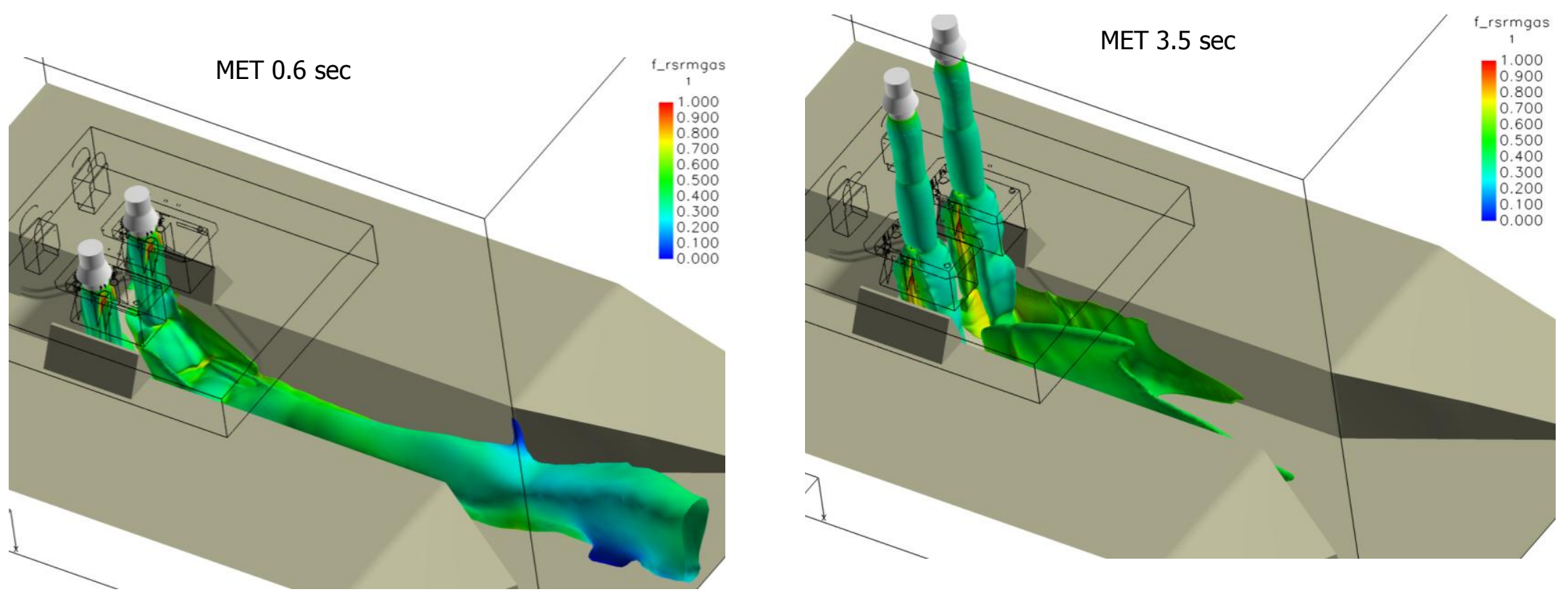


\section{Designing SLS for Liftoff Environment}

- Existing IOP prediction methods developed for Space Shuttle may not be capable of accurately modeling impact on the SLS vehicle due to the much different configuration: Placement of SRB plumes close to core engines inside single exhaust hole

- CFD taking important role in characterization of launch environment for SLS: provide guidance to the redesign efforts of mobile launcher, flame trench main deflector, and placement of sound suppression water

- CFD provides early design guidance in the layout of the liftoff configuration for optimal acoustic load mitigation

- Concept of identifying regions of noise sources in the CFD simulations and applying targeted placement of water injection aimed at those regions will be used as a potent design tool for designing sound suppression water systems

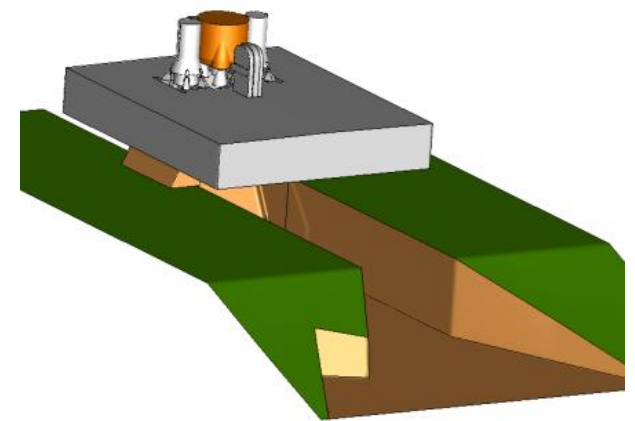

Example of SLS launch pad configuration CFD model

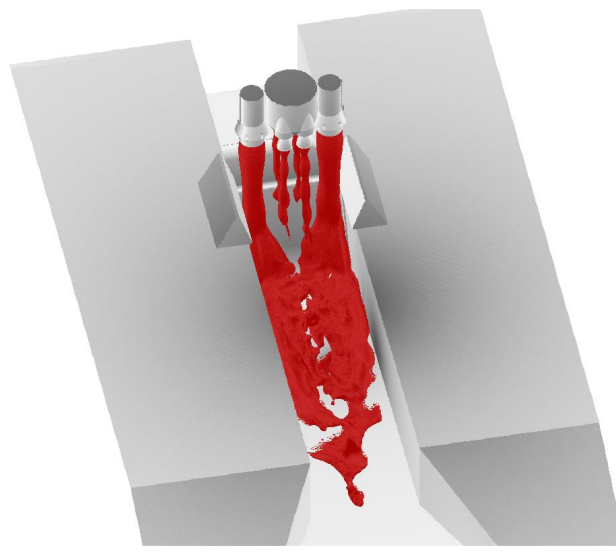

Multiple engine plume mass flow envelope

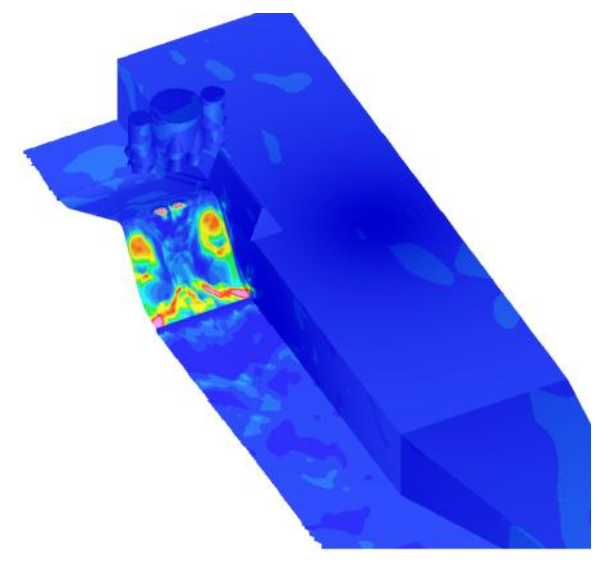

Flame deflector surface impingement pressure loads 


\section{SLS Ignition Overpressure Simulations}

- $\quad$ CFD transient simulations of SRB start-up applied in ongoing SLS Design Analysis Cycles

- Supports configuration design of mobile launcher opening and flame deflector in flame trench to mitigate liftoff loads on vehicle and launch facility
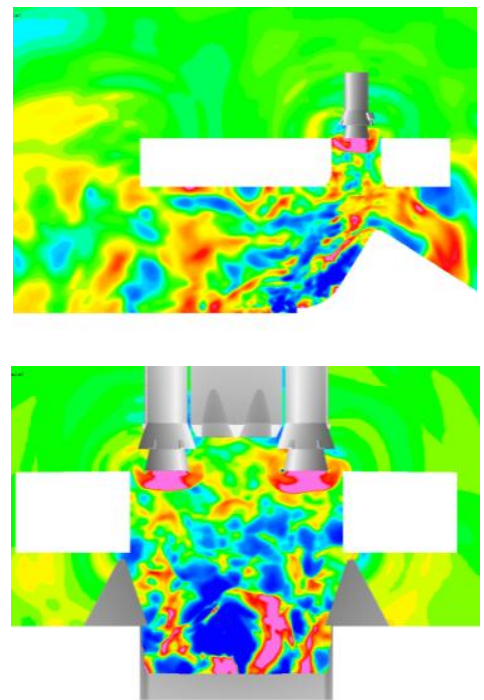

SRB nozzle flow ramp-up into fully developed SSME flowfield
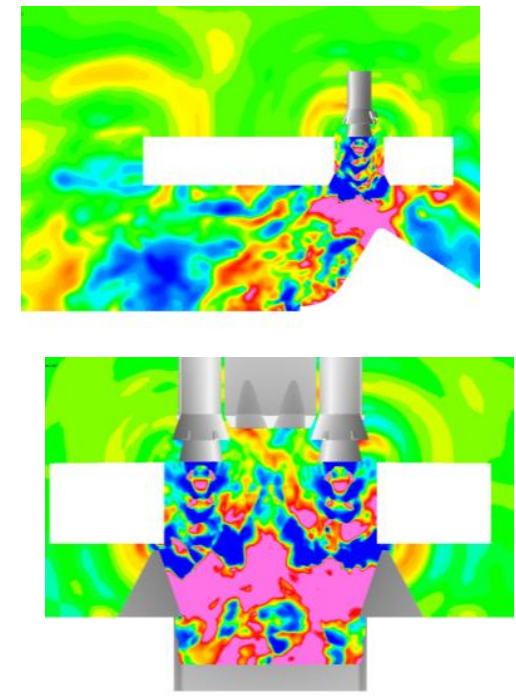

Formation of IOP source under launch pad
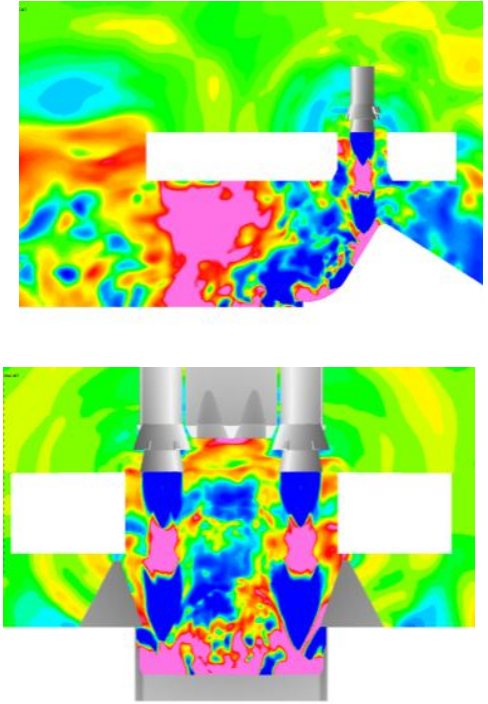

Propagation of duct overpressure wave

Ignition overpressure field simulation for SLS launch pad in cutting plane through SRBS

\section{JACOBS}




\section{Extend CFD Analysis to Liftoff Acoustic}

- Examples demonstrate Loci/CHEM CFD tool offers validated high-fidelity physics capability

- Mature CFD modeling capability is in place to capture many dominant liftoff plume flow environment features

- Desired next step to apply this multi-physics capability to also tackle effects on launch acoustic environment

- Important to retain complex physics feature modeling capability since they also drive acoustic field characteristics

- Multiple plumes with different plume gas composition (directivity)

- Plume impingement, spillage turbulence modeling (new sources)

- Turbulent plume mixing, requiring LES (Mach wave noise)

- Plume characteristics under launch pad and in flame trench

- Water deluge multi-phase effects on turbulent (acoustic) energy

- Problem: CFD too dissipative for acoustic field propagation

- CFD numerics are $2^{\text {nd }}$ order time, $2^{\text {nd }}$ order space accurate but not low dispersion/low dissipation

- Mesh resolution for preserving farfield acoustics for frequencies of interest (up to $5 \mathrm{kHz}$ ) in liftoff domain (100's of meters) exceeds foreseeable computing resources 


\section{CFD Based Liftoff Acoustic Modeling}

Two avenues towards high-fidelity CFD based liftoff acoustics predictions

- Long-term approach: Improve CFD algorithms beyond current $2^{\text {nd }}$ order accuracy

- Higher order spatial and temporal accuracy

- Lower numerical dissipation schemes

- Difficult to achieve while retaining multi-species, reacting, turbulent plume flow important for capturing acoustic sources

- Near term approach: Implement hybrid approach of CFD + CAA

- Utilize existing plume modeling fidelity to capture acoustic sources originating from plumes, impingement, water suppression effects

- Existing CFD modeling features important physics (multi-phase plume, turbulence, LES, gas-water phase effects from deluge, etc.) but too dissipative

- Propagate CAA from source surfaces enveloping noise source regions 


\section{Noise Surface Enclosure Definition}

- Various physical parameters considered in attempts to clearly identify regions of noise sources in a CFD flowfield: Turbulent kinetic energy, Reynolds stresses, velocity divergence, vorticity $Q$ function, ...

- In current work, the most physically intuitive and insightful method of identifying noise source regions was obtained by visualizing the magnitude of the Lighthill stress tensor

- $\quad$ Starting from first principle: Lighthill Acoustic Analogy

- Lighthill Equation

$$
\frac{\partial^{2} \rho^{\prime}}{\partial t^{2}}-c_{0}^{2} \frac{\partial^{2} \rho^{\prime \prime}}{\partial x_{i}^{2} !}=\frac{\partial^{2} T_{i j}}{\partial x_{i} \partial x_{j}}
$$

- Lighthill stress tensor $T_{i j}=\rho u_{i} u_{j}+\boldsymbol{\phi}-p_{0}-c_{0} \boldsymbol{b}-\rho_{0} \delta_{i j} \stackrel{1}{\jmath} \tau_{i j}$

- Reduces to

$$
T_{i j} \approx \rho u_{i} u_{j} \quad \text { (Other terms negligible) }
$$

- Envelope of Lighthill Stress Tensor Magnitude can now be used to identify acoustic source regions 


\section{Nasi Example of Noise Surface Enclosure Definition}

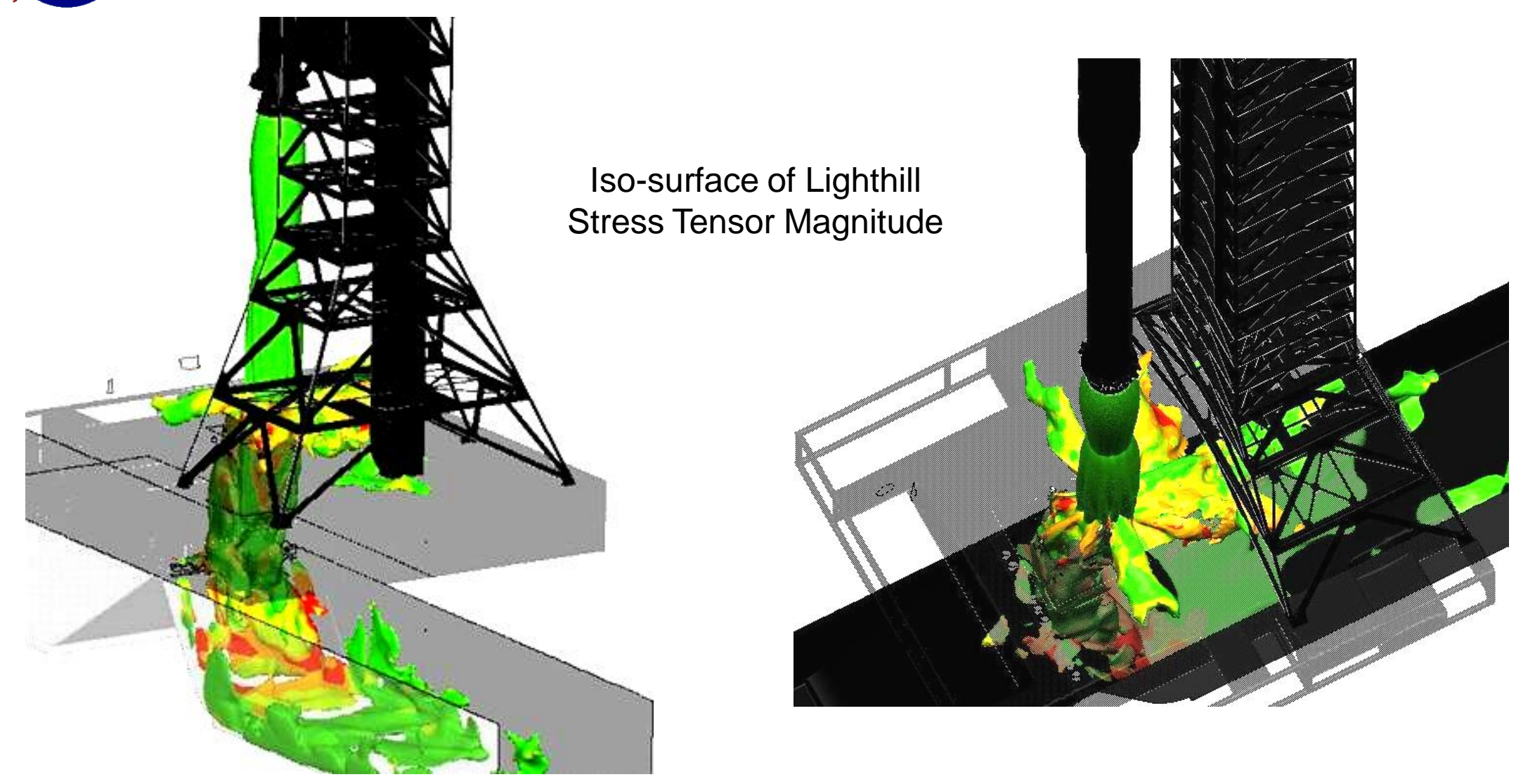

- Visualization of Noise Sources for ASMAT Plume Impingement

- Considerable challenge remains in defining water-tight envelope of source regions

\section{JACOBS}




\section{Utilize Knowledge Of Noise Source Regions}

- Identification of noise source region can be used in two important ways for prediction and mitigation of liftoff acoustics:

- First, a geometrically simplified boundary surface can be defined that engulfs all acoustics sources near the pad

- The initial condition inputs for a farfield acoustic field propagation method can then be extracted from the CFD simulation over this surface.

- Extraction allows for a bridge between CFD and CAA simulations and development of a coupled, hybrid CFD/CAA methodology which can quantify the acoustic loads on the full vehicle.

- Secondly, visualization of the extent and location of the noise source regions can be used for pinpointing mitigation measures using CFD.

- The effect of placing sound suppression water jets strategically aimed at noise source regions on the launch pad can be immediately visualized in changes in the Lighthill source regions.

- Enables CFD to become a targeted engineering design tool for sound suppression measures.

- Measures include the placement and mass flow specification of sound suppression water jets as well as modifications to the launch pad structure geometry. 


\section{Farfield CAA for Liftoff Acoustic Loads}

- Complex plume path (through mobile launch ducts, deflection under ML, plume exiting flame trench)

- Reflection, diffraction, attenuation of acoustic waves on ML and tower structures

- BEM identified as most suitable for capturing complex launch pad topology effects

- Evaluated implementation of BEM method for hybrid CFD/CAA

- Facilitated through NASA Small Business Technology Transfer project (STTR) to utilize technology from existing software: FastBEM Acoustics (Prof. Liu, U. Cincinnati)

- FastBEM Solves Helmholtz Equation via Boundary Integral Equation (BIE) Form

- Fast multi-pole BEM for solving 3-D, interior/exterior, radiation/scattering problems with velocity, pressure and impedance BCs

- Fast Multi-pole Method (FMM) reduces the cost (CPU time \& storage) for BEM to $O(M)$

- Demonstrated fast and accurate wideband acoustic analysis from low to high frequencies without compromising the BEM model size and accuracy

- Large-scale acoustic BEM models with unknowns (DOFs) up to several millions solved on PCs and even larger models on supercomputers

\section{JACOBS}




\section{Liftoff CFD-CAA Process Implementation}

- Establishing process to extract surface enclosing acoustic noise source regions

- Complicated enclosure surfaces result from plume interaction with structures

- Lighthill stress tensor visualization approach looks promising

- Developing handover process of acoustic enclosure surface to FastBEM input BC utilizing existing Loci/CHEM native post-processing tools

- Identifying FastBEM software modifications and improvements for NASA specific applications

- Large physical domain, complex enclosed structures topology

- Port software to NASA supercomputer facilities
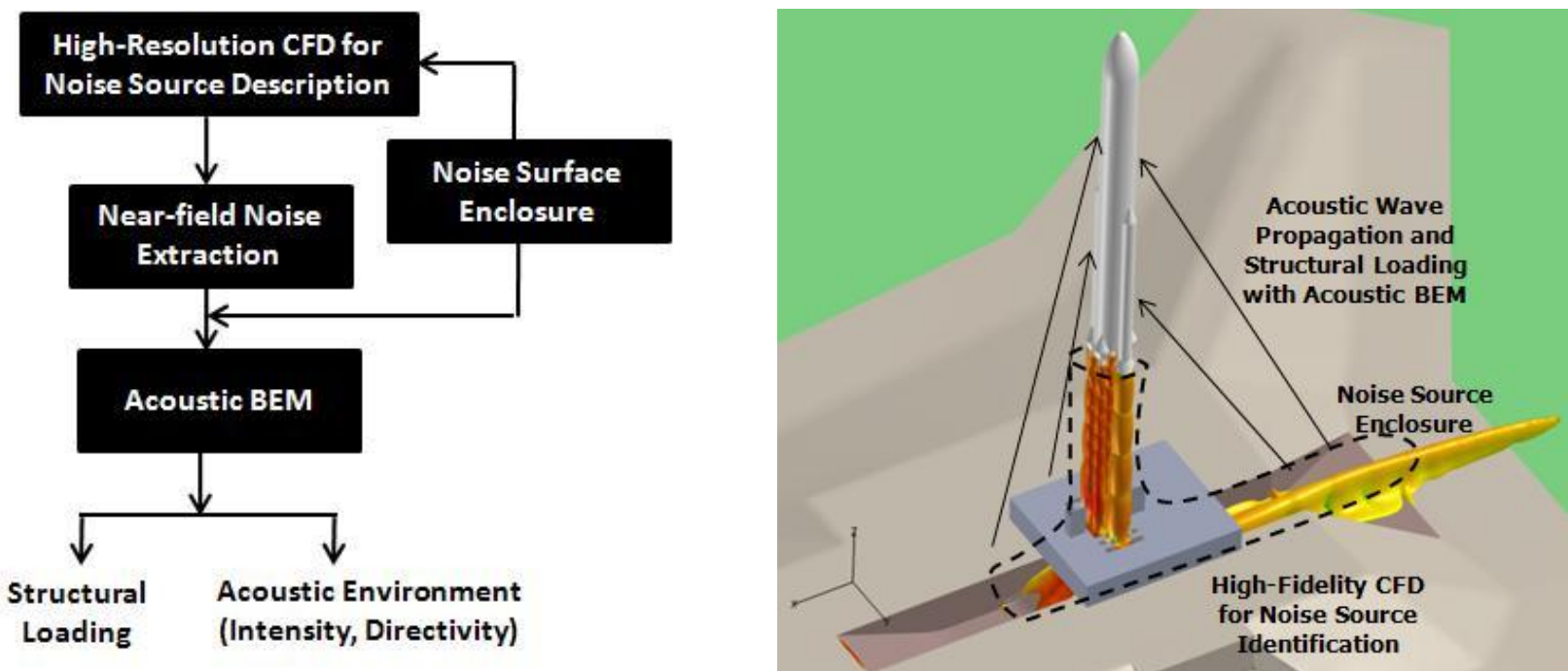


\section{Conclusions}

- MSFC Fluid Dynamics Branch responsible for NASA liftoff environments

- Sophisticated, validated CFD analysis capability in place for liftoff flow environments (pad and flame trench aerothermal, IOP, debris transport, ...)

- Now extending CFD analysis to predict SLS liftoff environments

- Intent is to apply CFD to capture effects not included in empirical liftoff acoustic methodologies: plume impingement, flame trench plume interaction, water deluge, ...

- These capabilities now offer the possibility of analysis support for the geometric design of the launch pad, flame trench, and targeted placement of water injection systems for sound suppression

- Hybrid CFD-CAA approach being pursued to predict farfield vehicle acoustic loads

- Progress in identifying acoustic sources regions in complex launch pad topology by utilizing first principle Lighthill stress tensor visualization

- Testing BEM approach for hybrid CFD+CAA because of capability to capture interaction with complex launch pad topology 